JOURNAL OF THE

AMERICAN MATHEMATICAL SOCIETY

Volume 24, Number 1, January 2011, Pages 51-80

S 0894-0347(2010)00680-7

Article electronically published on September 8, 2010

\title{
HECKE FIELDS OF ANALYTIC FAMILIES OF MODULAR FORMS
}

\author{
HARUZO HIDA
}

Fix a prime $p$, and put $\mathbf{p}=4$ if $p=2$ and $\mathbf{p}=p$ otherwise. For a Hecke eigenform $f \in S_{k}\left(\Gamma_{0}\left(N p^{r} \mathbf{p}\right), \psi\right)(p \nmid N, r \geq 0)$ and a subfield $K$ of $\mathbb{C}$, the Hecke field $K(f)$ inside $\mathbb{C}$ is generated over $K$ by the eigenvalues $a_{n}=a(n, f)$ of $f$ for the Hecke operators $T(n)$ for all $n$. Then $\mathbb{Q}(f)$ is a finite extension of $\mathbb{Q}$ sitting inside the algebraic closure $\overline{\mathbb{Q}}$ in $\mathbb{C}$. Let $\Gamma=1+\mathbf{p} \mathbb{Z}_{p}$, which is a maximal torsion-free subgroup of $\mathbb{Z}_{p}^{\times}$. We choose and fix a generator $\gamma:=(1+\mathbf{p}) \in \Gamma$ so that $\Gamma=\gamma^{\mathbb{Z}_{p}}$ and identify the Iwasawa algebra $\Lambda=W[[\Gamma]]$ with the power series ring $W[[x]]$ by $\Gamma \ni \gamma \mapsto(1+x)$ (for a discrete valuation ring $W$ finite flat over $\mathbb{Z}_{p}$ ). A $p$-adic slope 0 analytic family of eigenforms $\mathcal{F}=\left\{f_{P} \mid P \in \operatorname{Spec}(\mathbb{I})\left(\mathbb{C}_{p}\right)\right\}$ is indexed by points of $\operatorname{Spec}(\mathbb{I})\left(\mathbb{C}_{p}\right)$, where $\operatorname{Spec}(\mathbb{I})$ is a finite flat irreducible covering of $\operatorname{Spec}(\Lambda)$. For each $P \in \operatorname{Spec}(\mathbb{I}), f_{P}$ is a $p$-adic modular form of slope 0 of level $N p^{\infty}$ for a fixed prime to $p$-level $N$. The family is called analytic because $P \mapsto a\left(n, f_{P}\right)$ is a $p$-adic analytic function on $\operatorname{Spec}(\mathbb{I})$. We call $P$ arithmetic of weight $k=k(P) \in \mathbb{Z}$ with character $\varepsilon_{P}: \Gamma \rightarrow \mu_{p^{\infty}}\left(\mathbb{C}_{p}\right)$ if $P$ contains $\left(1+x-\varepsilon_{P}(\gamma) \gamma^{k}\right) \in \Lambda$ and $k(P) \geq 2$. If $P$ is arithmetic, $f_{P}$ is known to be a $p$-stabilized classical Hecke eigenform and has Neben character $\psi_{P}$ whose restriction to $\Gamma$ is given by $\varepsilon_{P}$. We write $p^{r(P)}$ for the order of $\varepsilon_{P}$ (then, the classical form $f_{P}$ has level $N p^{r(P)} \mathbf{p}$ ). In order to make the introduction succinct, we put off, to Section 11 recalling the theory of analytic families of eigenforms including the definition and necessary properties of $\mathrm{CM}$ families. We define the following Hecke fields out of $\mathcal{F}$ :

$(\mathrm{V})$ For a fixed level $N p^{r} \mathbf{p}(0 \leq r \leq \infty), \mathbb{Q}_{V, r}(\mathcal{F})$ is the composite of $\mathbb{Q}\left(f_{P}\right)$ for all arithmetic $P \in \operatorname{Spec}(\mathbb{I})\left(\overline{\mathbb{Q}}_{p}\right)$ with $k(P) \geq 2$ and $\varepsilon_{P}$ factoring through $\Gamma / \Gamma^{p^{r}}$.

(H) For a fixed weight $k \geq 2, \mathbb{Q}_{H, k}(\mathcal{F})$ is the composite of $\mathbb{Q}\left(f_{P}\right)$ for all arithmetic $P \in \operatorname{Spec}(\mathbb{I})\left(\overline{\mathbb{Q}}_{p}\right)$ with $k(P)=k$.

Here the composite is taken in the algebraic closure $\overline{\mathbb{Q}}$ inside $\mathbb{C}$. If $r=0$, periodically in $k(P), f_{P}$ is old at $p$ associated to a unique new form $f_{P}^{\circ}$ of level $N$ prime to $p$; so, putting $\mathcal{F}^{\circ}=\left\{f_{P}^{\circ}\right\}$ for such arithmetic $P$ s, we can also define $\mathbb{Q}_{V}\left(\mathcal{F}^{\circ}\right)$ as the composite of $\mathbb{Q}\left(f_{P}^{\circ}\right)$. Abusing the notation, we put $\mathbb{Q}_{V,-1}(\mathcal{F}):=\mathbb{Q}_{V}\left(\mathcal{F}^{\circ}\right)$.

\footnotetext{
Received by the editors June 19, 2009 and, in revised form, February 8, 2010, and April 29, 2010 .

2010 Mathematics Subject Classification. Primary 11E16, 11F11, 11F25, 11F27, 11F30, 11F33, $11 \mathrm{~F} 80$.

The author is partially supported by the NSF grant: DMS 0753991 and DMS 0854949, and part of this work was done during the author's stay in January to March 2010 at the Institut Henri Poincaré - Centre Emile Borel. The author thanks this institution for its hospitality and support.
} 
Once L. Clozel asked me if (or when) the Hecke field $\mathbb{Q}_{V, r}(\mathcal{F})$ for a finite $r$ is a finite extension of $\mathbb{Q}$. At the time, for a lack of examples, my answer was "probably" that it is finite if and only if the family contains a CM theta series (i.e., a binary theta series) of weight $k(P) \geq 2$. If the family contains a binary theta series of weight $k \geq 2$ of an imaginary quadratic field $M$, all forms have CM by the same (fixed) imaginary quadratic field $M$, and hence $\mathbb{Q}_{V, r}(\mathcal{F})(r<\infty)$ is contained in a finite extension of the imaginary quadratic field $M$ (see Corollary 4.2). The following conjecture is anybody's guess (and perhaps Clozel must have had the same thought):

Conjecture. The following three assertions are equivalent:

(1) The field $\mathbb{Q}_{V, r}(\mathcal{F})(r<\infty)$ is a finite extension of $\mathbb{Q}$.

(2) The family $\mathcal{F}$ contains a theta series of weight $k \geq 2$ of an imaginary quadratic field $M$.

(3) The family $\mathcal{F}$ is made up of theta series of a fixed imaginary quadratic field $M$.

As already mentioned, the assertions (2) and (3) are equivalent; so, hereafter, we only state (2), which implies (3). We tentatively recall that $\mathcal{F}$ has $\mathrm{CM}$ (complex multiplication) by $M$ if the above (2) is satisfied (see (CM1-3) in Section 1 for a precise definition of $\mathrm{CM}$ families). When $N=1$ and $r=-1$, Maeda made a conjecture predicting that $d(P)=\left[\mathbb{Q}\left(f_{P}^{\circ}\right) ; \mathbb{Q}\right]=\operatorname{dim}_{\mathbb{C}} S_{k(P)}\left(S L_{2}(\mathbb{Z})\right)$ and the Galois group of the Galois closure of $\mathbb{Q}\left(f_{P}^{\circ}\right) / \mathbb{Q}$ is isomorphic to the symmetric group of $d(P)$ letters (see [HM, Conjecture 1.2]; see also Corollary 6.3 and Conjecture 8.1 in the text). Since there is no CM family of prime-to- $p$ level $N=1$, his conjecture implies our conjecture if $N=1$.

Write $A_{P}$ for the abelian variety (defined over $\mathbb{Q}$ ) associated to $f_{P}$ at an arithmetic point $P \in \operatorname{Spec}(\mathbb{I})$ with $k(P)=2$ and $r(P) \leq r$ (defined by Shimura IAT, Theorem 7.14]). Our result towards the conjecture is as follows.

Vertical Theorem. Suppose $p>2$ and that we have an arithmetic point $P \in$ $\operatorname{Spec}(\mathbb{I})$ of weight $k(P)=2$ with $r(P) \leq r$ such that $A_{P}$ has good ordinary reduction over $\mathbb{Z}_{p}\left[\mu_{p^{r+1}}\right]$. Then the field $\mathbb{Q}_{V, r}(\mathcal{F})$ for a bounded p-power level $N p^{r+1}(r \geq 0)$ is a finite extension of $\mathbb{Q}$ if and only if $\mathcal{F}$ contains a theta series of weight $k \geq 2$ of an imaginary quadratic field.

The abelian variety $A_{P}$ has good ordinary reduction at $p$ if one of the following three conditions is satisfied (see Corollary 2.2 in the text and [GME, Section 4.2]):

- $A_{P}$ is an elliptic curve with good reduction at $p$,

- $A_{P}$ has good reduction at $p$ and $\mathbb{Q}\left(f_{P}\right) / \mathbb{Q}$ is unramified at $p$,

- $A_{P}$ has good reduction at $p$ and $a\left(p, f_{P}\right)$ generates the integral closure of $\mathbb{Z}_{p}$ in $\mathbb{Q}\left(f_{P}\right) \otimes_{\mathbb{Z}} \mathbb{Z}_{p}$

The main point of the assumption is the "ordinarity" (not really the good reduction), and we analyze closely the case where $A_{P}$ has multiplicative reduction at $p$ in Corollary 7.3 . By the above facts, for any family $\mathcal{F}$ giving a non-CM elliptic curve of ordinary good reduction at $p, \mathbb{Q}_{V, r}(\mathcal{F})$ is an infinite extension. In the text, we prove a stronger version of the theorem (Theorem 3.2) telling us that any non-CM family $\mathcal{F}$ having an arithmetic $P$ associated to an "ordinary" motive potentially crystalline (at $p$ ) has infinite $\mathbb{Q}_{V, r}(\mathcal{F})$; so, if $\mathbb{Q}\left(f_{P}^{\circ}\right)=\mathbb{Q}$ with $k(P)>2$, $\mathbb{Q}_{V, r}(\mathcal{F})$ has infinite degree over $\mathbb{Q}$. In particular, for the $p$-adic family $\mathcal{F}_{\Delta}$ containing Ramanujan's $\Delta$-function, we have $\left[\mathbb{Q}_{V, r}\left(\mathcal{F}_{\Delta}\right): \mathbb{Q}\right]=\infty$ (though we do not know 
if $\mathcal{F}_{\Delta}$ always has a weight 2 point $P$ with $A_{P}$ having ordinary good reduction). For a non-CM family $\mathcal{F}$, we conjecture that $\lim \sup _{P, f_{P} \in \mathcal{F}}\left[\mathbb{Q}\left(f_{P}\right): \mathbb{Q}\right]=\infty$ for $P$ running over arithmetic points $P$ of bounded level (Conjecture 8.1). If we do not bound the level exponent $r(P), \lim _{\sup _{P, f_{P} \in \mathcal{F}}}\left[K\left(f_{P}\right): K\right]=\infty$ for $K=\mathbb{Q}\left(\mu_{p^{\infty}}\right)$ as in the following theorem (see also Corollary 6.3).

There is a horizontal version. We have $T(l)^{2}-T\left(l^{2}\right)=l \cdot T(l, l)$ for primes $l$ outside the level (for the central action $T(l, l)$ in [AT, Theorem 3.24]), and $f_{P} \mid T(l, l)=$ $\varepsilon_{P}(l) l^{k-2} f_{P}$ if $l \in \Gamma$. Thus $\mathbb{Q}_{H, k}(\mathcal{F})$ contains $\mathbb{Q}\left(\mu_{p} \infty\right)$. Again, if the family contains a CM theta series of weight $k \geq 2$ of a quadratic field $M$, all forms have CM by the same imaginary quadratic field $M$, and hence $\mathbb{Q}_{H, k}(\mathcal{F})(k \geq 2)$ is contained in a finite extension of $M\left(\mu_{p^{\infty}}\right)$ (Corollary 4.2). The horizontal theorem is more complete as it does not require the existence of $P$ with ordinary $A_{P}$.

Horizontal Theorem. Suppose $p>2$. The Hecke field $\mathbb{Q}_{H, k}(\mathcal{F})$ for a fixed weight $k \geq 2$ is a finite extension of $K:=\mathbb{Q}\left(\mu_{p}\right)$ if and only if $\mathcal{F}$ contains a theta series of weight $k \geq 2$ of an imaginary quadratic field. Moreover, for a non-CM family $\mathcal{F}$, we have $\lim \sup _{P, f_{P} \in \mathcal{F}}\left[K\left(f_{P}\right): K\right]=\infty$ for $P$ running over infinitely many arithmetic points $P$ of fixed weight (i.e., $k(P)=k \geq 2$ ).

In this paper, we study these assertions and their variants. We will prove a stronger version of the horizontal theorem (Theorem 3.3) which implies not only the horizontal theorem but also the vertical theorem. The proof of the above theorems is based on the elementary finiteness of Weil $l$-numbers of given weight in $\mathbb{Q}\left[\mu_{p} \infty\right]$ up to multiplication by roots of unity and on a simple analysis of the prime factorization of Weil numbers in Hecke fields. The same finiteness of Weil $l$-numbers of given weight (up to roots of unity) in the maximal abelian extension of $\mathbb{Q}$ is a nontrivial fact (a conjecture of Robinson proven by Loxton [Lo; see Lemma 2.3 in the text).

The author owes much to Kiran Kedlaya who supplied us with a proof of Lemma 5.1 more elementary than the one by the author and informed the author about the result of Loxton. The author would like to thank him for his assistance.

\section{Contents}

1. Analytic families of Hecke eigenforms 53

2. Weil numbers 56

3. Theorems and conjectures 61

4. Hecke fields and their quadratic extensions 63

5. Results towards the strong horizontal theorem 66

6. Proof of the strong horizontal theorem 71

7. Results towards vertical conjectures

8. Proof of vertical theorems 78

References $\quad 79$

\section{Analytic families of HeCke eigenforms}

Fix a field embedding $\overline{\mathbb{Q}} \stackrel{i_{p}}{\hookrightarrow} \overline{\mathbb{Q}}_{p} \subset \mathbb{C}_{p}$ and a positive integer $N$ prime to $p$. Here $\overline{\mathbb{Q}}$ is the algebraic closure of $\mathbb{Q}$ in $\mathbb{C}$ and $\overline{\mathbb{Q}}_{p}$ is an algebraic closure of $\mathbb{Q}_{p}$. A $p$-adic 
analytic family $\mathcal{F}$ of modular forms is defined with respect to the fixed embedding $i_{p}: \overline{\mathbb{Q}} \hookrightarrow \mathbb{C}_{p}$. We write $|\alpha|_{p}$ for the $p$-adic absolute value (with $|p|_{p}=1 / p$ ) induced by $i_{p}$. We also fix a field embedding $\overline{\mathbb{Q}}_{p} \hookrightarrow \mathbb{C}$ inducing the inclusion on $\overline{\mathbb{Q}} \subset \mathbb{C}$. As a base ring, we take a (sufficiently large) discrete valuation ring $W \subset \overline{\mathbb{Q}}_{p}$ finite flat over the $p$-adic integer ring $\mathbb{Z}_{p}$. Recall that $\mathbf{p}=p$ if $p$ is odd and $\mathbf{p}=4$ otherwise. Take a Dirichlet character $\psi:\left(\mathbb{Z} / N p^{r} \mathbf{p} \mathbb{Z}\right)^{\times} \rightarrow W^{\times}$with $(p \nmid N, r \geq 0)$, and consider the space of elliptic cusp forms $S_{k}\left(\Gamma_{0}\left(N p^{r} \mathbf{p}\right), \psi\right)$ with character $\psi$ as defined in [IAT, (3.5.4)]. Let the ring $\mathbb{Z}[\psi] \subset \mathbb{C}$ and $\mathbb{Z}_{p}[\psi] \subset \overline{\mathbb{Q}}_{p}$ be generated by the values $\psi$ over $\mathbb{Z}$ and $\mathbb{Z}_{p}$, respectively. The Hecke algebra over $\mathbb{Z}[\psi]$ is the subalgebra of the linear endomorphism algebra of $S_{k}\left(\Gamma_{0}\left(N p^{r} \mathbf{p}\right), \psi\right)$ generated by Hecke operators $T(n)$ :

$$
h=\mathbb{Z}[\psi][T(n) \mid n=1,2, \cdots] \subset \operatorname{End}\left(S_{k}\left(\Gamma_{0}\left(N p^{r} \mathbf{p}\right), \psi\right)\right),
$$

where $T(n)$ is the Hecke operator as in [AT, §3.5]. We put $h_{k, \psi}=h_{k, \psi / W}=$ $h \otimes_{\mathbb{Z}[\psi]} W$. When we need to indicate that our $T(l)$ is the Hecke operator of a prime factor $l$ of $N p^{r} \mathbf{p}$, we write it as $U(l)$, since $T(l)$ acting on a subspace $S_{k}\left(\Gamma_{0}\left(N^{\prime}\right), \psi\right) \subset S_{k}\left(\Gamma_{0}\left(N p^{r} \mathbf{p}\right), \psi\right)$ of level $N^{\prime}$ prime to $l$ does not coincide with $U(l)$ on $S_{k}\left(\Gamma_{0}\left(N p^{r} \mathbf{p}\right), \psi\right)$. The ordinary part $\mathbf{h}_{k, \psi / W} \subset h_{k, \psi / W}$ is the maximal ring direct summand on which $U(p)$ is invertible. We write $e$ for the idempotent of $\mathbf{h}_{k, \psi / W}$, and hence $e=\lim _{n \rightarrow \infty} U(p)^{n !}$ under the $p$-adic topology of $h_{k, \psi / W}$. By the fixed embedding $\overline{\mathbb{Q}}_{p} \hookrightarrow \mathbb{C}$, the idempotent $e$ not only acts on the space of modular forms with coefficients in $W$ but also on the classical space $S_{k}\left(\Gamma_{0}\left(N p^{r} \mathbf{p}\right), \psi\right)$. We write the image of the idempotent as $S_{k}^{\text {ord }}$.

Fix $\psi$, and assume now that $\psi_{p}=\left.\psi\right|_{\mathbb{Z}_{p}^{\times}}$has conductor at most $\mathbf{p}$ and $\psi(-1)=$ 1. Let $\omega$ be the modulo $\mathbf{p}$ Teichmüller character (so, if $p=2, \omega$ is the unique non-trivial character of $\left.(\mathbb{Z} / 4 \mathbb{Z})^{\times}\right)$. Recall that the multiplicative group $\Gamma:=1+$ $\mathbf{p} \mathbb{Z}_{p}$ in $\mathbb{Z}_{p}^{\times}$and its topological generator $\gamma=1+\mathbf{p}$. Then the Iwasawa algebra $\Lambda=W[[\Gamma]]=\lim _{n} W\left[\Gamma / \Gamma^{p^{n}}\right]$ is identified with the power series ring $W[[x]]$ by a $W$-algebra isomorphism sending $\gamma \in \Gamma$ to $1+x$. As constructed in [H86a, H86b and GME, we have a unique 'big' ordinary Hecke algebra $\mathbf{h}$. The algebra $\mathbf{h}$ is characterized by the following two properties (called Control theorems; see [H86a, Theorem 3.1, Corollary 3.2] and [H86b, Theorem 1.2] for $p \geq 5$ and [GME, Theorem 3.2.15 and Corollary 3.2.18] for general $p$ ):

(C1) $\mathbf{h}$ is free of finite rank over $\Lambda$ equipped with $T(n) \in \mathbf{h}$ for all $1 \leq n \in \mathbb{Z}$ (so $U(l)$ for $l \mid N p)$,

(C2) if $k \geq 2$ and $\varepsilon: \mathbb{Z}_{p}^{\times} \rightarrow \mu_{p \infty}$ is a character,

$$
\mathbf{h} /\left(1+x-\varepsilon(\gamma) \gamma^{k}\right) \mathbf{h} \cong \mathbf{h}_{k, \varepsilon \psi_{k}}(\gamma=1+p) \text { for } \psi_{k}:=\psi \omega^{-k}
$$

sending $T(n)$ to $T(n)$ (and $U(l)$ to $U(l)$ for $l \mid N p$ ).

In the sequel, we often make use of another variable $X=\gamma^{-1}(1+x)-1$. We still have $\Lambda=W[[X]]$. The prime ideal $((1+x)-\gamma)$ is equal to $(X)$; so, if $(\mathrm{C} 2)$ were valid for $k=1, \mathbf{h} / X \mathbf{h}$ would have been the Hecke algebra of weight 1 ; so, we call the variable $X$ of $\Lambda$ the variable centered at weight 1 .

Let $\operatorname{Spec}(\mathbb{I})$ be a reduced irreducible component $\operatorname{Spec}(\mathbb{I}) \subset \operatorname{Spec}(\mathbf{h})$. Write $a(n)$ for the image of $T(n)$ in $\mathbb{I}$ (so, $a(p)$ is the image of $U(p)$ ). If a point $P$ of $\operatorname{Spec}(\mathbb{I})\left(\overline{\mathbb{Q}}_{p}\right)$ kills $\left(1+x-\varepsilon(\gamma) \gamma^{k}\right)=\left(1+X-\varepsilon(\gamma) \gamma^{k-1}\right)$ with $2 \leq k \in \mathbb{Z}$ (i.e., 
$\left.P\left(\left(1+x-\varepsilon(\gamma) \gamma^{k}\right)\right)=0\right)$, we call it an arithmetic point and we write $\varepsilon_{P}=\varepsilon$, $k(P)=k \geq 2$ and $p^{r(P)}$ for the order of $\varepsilon_{P}$. If $P$ is arithmetic, by (C2), we have a Hecke eigenform $f_{P} \in S_{k}\left(\Gamma_{0}\left(N p^{r(P)} \mathbf{p}\right), \varepsilon \psi_{k}\right)$ such that its eigenvalue for $T(n)$ is given by $a_{P}(n):=P(a(n)) \in \overline{\mathbb{Q}}_{p}$ for all $n$. Thus $\mathbb{I}$ gives rise to a family $\mathcal{F}=\left\{f_{P} \mid\right.$ arithemtic $\left.P \in \operatorname{Spec}(\mathbb{I})\right\}$ of Hecke eigenforms. We define a $p$-adic analytic family of slope 0 (with coefficients in $\mathbb{I}$ ) to be the family as above of Hecke eigenforms associated to an irreducible component $\operatorname{Spec}(\mathbb{I}) \subset \operatorname{Spec}(\mathbf{h})$. We call this family slope 0 because $\left|a_{P}(p)\right|_{p}=1$ for the $p$-adic absolute value $|\cdot|_{p}$ of $\overline{\mathbb{Q}}_{p}$ (it is also often called an ordinary family). We call this family analytic because the Hecke eigenvalue $a_{P}(n)$ for $T(n)$ is given by an analytic function $a(n)$ on (the rigid analytic space associated to) the $p$-profinite formal spectrum $\operatorname{Spf}(\mathbb{I})$. Identify $\operatorname{Spec}(\mathbb{I})\left(\overline{\mathbb{Q}}_{p}\right)$ with $\operatorname{Hom}_{W \text {-alg }}\left(\mathbb{I}, \overline{\mathbb{Q}}_{p}\right)$ so that each element $a \in \mathbb{I}$ gives rise to a "function" $a: \operatorname{Spec}(\mathbb{I})\left(\overline{\mathbb{Q}}_{p}\right) \rightarrow \overline{\mathbb{Q}}_{p}$ whose value at $\left(P: \mathbb{I} \rightarrow \overline{\mathbb{Q}}_{p}\right) \in \operatorname{Spec}(\mathbb{I})\left(\overline{\mathbb{Q}}_{p}\right)$ is $a_{P}:=P(a) \in \overline{\mathbb{Q}}_{p}$. Then $a$ is an analytic function of the rigid analytic space associated to $\operatorname{Spf}(\mathbb{I})$. Taking a finite covering $\operatorname{Spec}(\widetilde{\mathbb{I}})$ of $\operatorname{Spec}(\mathbb{I})$ with surjection $\operatorname{Spec}(\widetilde{\mathbb{I}})\left(\overline{\mathbb{Q}}_{p}\right) \rightarrow \operatorname{Spec}(\mathbb{I})\left(\overline{\mathbb{Q}}_{p}\right)$, abusing slightly the definition, we may regard the family $\mathcal{F}$ as being indexed by arithmetic points of $\operatorname{Spec}(\widetilde{\mathbb{I}})\left(\overline{\mathbb{Q}}_{p}\right)$, where arithmetic points of $\operatorname{Spec}(\widetilde{\mathbb{I}})\left(\overline{\mathbb{Q}}_{p}\right)$ are made up of the points above arithmetic points of $\operatorname{Spec}(\mathbb{I})\left(\overline{\mathbb{Q}}_{p}\right)$. The choice of $\widetilde{\mathbb{I}}$ is often the normalization of $\mathbb{I}$ or the integral closure of $\mathbb{I}$ in a finite extension of the quotient field of $\mathbb{I}$.

Each (reduced) irreducible component $\operatorname{Spec}(\mathbb{I}) \subset \operatorname{Spec}(\mathbf{h})$ has a 2-dimensional semi-simple (absolutely irreducible) continuous representation $\rho_{\mathbb{I}}$ of $\operatorname{Gal}(\overline{\mathbb{Q}} / \mathbb{Q})$ with coefficients in the quotient field of $\mathbb{I}$ (see $[\mathrm{H} 86 \mathrm{~b}]$ ). The representation $\rho_{\mathbb{I}}$ restricted to the $p$-decomposition group $D_{p}$ is reducible with unramified quotient character (e.g., GME, §4.2]). We write $\rho_{\mathbb{I}}^{s s}$ for its semi-simplification over $D_{p}$. As is well known now (e.g., GME, §4.2]), $\rho_{\mathbb{I}}$ is unramified outside $N p$ and satisfies, for primes $l \nmid N p$,

$$
\operatorname{Tr}\left(\rho_{\mathbb{I}}\left(F r o b_{l}\right)\right)=a(l), \rho_{\mathbb{I}}^{s s}\left(\left[\gamma^{s}, \mathbb{Q}_{p}\right]\right) \sim\left(\begin{array}{cc}
(1+X)^{s} & 0 \\
0 & 1
\end{array}\right), \rho_{\mathbb{I}}^{s s}\left(\left[p, \mathbb{Q}_{p}\right]\right) \sim\left(\begin{array}{cc}
* & 0 \\
0 & a(p)
\end{array}\right),
$$

where $\gamma^{s}=(1+\mathbf{p})^{s}=\sum_{n=0}^{\infty}\left(\begin{array}{l}s \\ n\end{array}\right) \mathbf{p}^{n} \in \mathbb{Z}_{p}^{\times}$for $s \in \mathbb{Z}_{p}$ and $\left[x, \mathbb{Q}_{p}\right]$ is the local Artin symbol.

By (Gal) and the Chebotarev density theorem, $\operatorname{Tr}\left(\rho_{\mathbb{I}}\right)$ has values in $\mathbb{I}$; so, $P$ o $\operatorname{Tr}\left(\rho_{\mathbb{I}}\right): \operatorname{Gal}(\overline{\mathbb{Q}} / \mathbb{Q}) \rightarrow \overline{\mathbb{Q}}_{p}\left(P \in \operatorname{Spec}(\mathbb{I})\left(\overline{\mathbb{Q}}_{p}\right)\right)$ gives rise to a pseudo-representation of Wiles (e.g., [MFG, §2.2]). Then by a theorem of Wiles, we can make a unique 2dimensional semi-simple continuous representation $\rho_{P}: \operatorname{Gal}(\overline{\mathbb{Q}} / \mathbb{Q}) \rightarrow G L_{2}\left(\overline{\mathbb{Q}}_{p}\right)$ unramified outside $N p$ with $\operatorname{Tr}\left(\rho_{P}\left(F r o b_{l}\right)\right)=a_{P}(l)$ for all primes $l$ outside $N p$ (though the construction of $\rho_{P}$ does not require the technique of pseudo-representation and was known before the invention of the technique; see [MW, §9, Proposition 1]). This is the Galois representation associated to the Hecke eigenform $f_{P}$ (constructed earlier by Shimura and Deligne) if $P$ is arithmetic (e.g., [GME, §4.2]).

A component $\mathbb{I}$ is called a $C M$ component if there exists a nontrivial character $\chi: \operatorname{Gal}(\overline{\mathbb{Q}} / \mathbb{Q}) \rightarrow \mathbb{I}^{\times}$such that $\rho_{\mathbb{I}} \cong \rho_{\mathbb{I}} \otimes \chi$. We also say that $\mathbb{I}$ has complex multiplication if $\mathbb{I}$ is a $\mathrm{CM}$ component. In this case, we call the corresponding family $\mathcal{F}$ a $\mathrm{CM}$ family (or we say that $\mathcal{F}$ has complex multiplication). If $\mathcal{F}$ is a $\mathrm{CM}$ family associated to $\mathbb{I}$ with $\rho_{\mathbb{I}} \cong \rho_{\mathbb{I}} \otimes \chi$, then $\chi$ is a quadratic character of $\operatorname{Gal}(\overline{\mathbb{Q}} / \mathbb{Q})$ which cuts out an imaginary quadratic field $M$, i.e., $\chi=\left(\frac{M / \mathbb{Q}}{}\right)$. Write $\widetilde{\mathbb{I}}$ for the integral closure of $\Lambda$ inside the quotient field of $\mathbb{I}$. The following three conditions 
are known to be equivalent:

(CM1) $\mathcal{F}$ has $C M$ with $\rho_{\mathbb{I}} \cong \rho_{\mathbb{I}} \otimes\left(\frac{M / \mathbb{Q}}{}\right)\left(\Leftrightarrow \rho_{\mathbb{I}} \cong \operatorname{Ind}_{M}^{\mathbb{Q}} \hat{\lambda}\right.$ for a character $\hat{\lambda}$ of $\operatorname{Gal}(\overline{\mathbb{Q}} / M)$ with values in $\left.\widetilde{\mathbb{I}}^{\times}\right)$.

(CM2) For all arithmetic $P$ of $\operatorname{Spec}(\mathbb{I})\left(\overline{\mathbb{Q}}_{p}\right), f_{P}$ is a binary theta series of the norm form of $M / \mathbb{Q}$.

(CM3) For some arithmetic $P$ of $\operatorname{Spec}(\mathbb{I})\left(\overline{\mathbb{Q}}_{p}\right), f_{P}$ is a binary theta series of the norm form of $M / \mathbb{Q}$.

Indeed, (CM1) is equivalent to $\rho_{\mathbb{I}} \cong \operatorname{Ind}_{M}^{\mathbb{Q}} \hat{\lambda}$ for a character $\widehat{\lambda}: \operatorname{Gal}(\overline{\mathbb{Q}} / M) \rightarrow$ $\widetilde{\mathbb{I}}^{\times}$unramified outside $N p$ (e.g., [MFG, Lemma 2.15]). Since the characteristic polynomial of $\rho_{\mathbb{I}}(\sigma)$ has coefficients in $\mathbb{I}$, its eigenvalues fall in $\widetilde{\mathbb{I}}$; so, the character $\widehat{\lambda}$ has values in $\widetilde{\mathbb{I}}^{\times}$(see H86c, Corollary 4.2]). Then by (Gal), $\widehat{\lambda}_{P}=P \circ \widehat{\lambda}$ : $\operatorname{Gal}(\overline{\mathbb{Q}} / M) \rightarrow \overline{\mathbb{Q}}_{p}^{\times}$for an arithmetic $P \in \operatorname{Spec}(\widetilde{\mathbb{I}})\left(\overline{\mathbb{Q}}_{p}\right)$ is a locally algebraic $p$-adic character, which is the $p$-adic avatar of a Hecke character $\lambda_{P}: M_{\mathbb{A}}^{\times} / M^{\times} \rightarrow \mathbb{C}^{\times}$of type $A_{0}$ of the quadratic field $M_{/ \mathbb{Q}}$. Then by the characterization (Gal) of $\rho_{\mathbb{I}}, f_{P}$ is the theta series with $q$-expansion $\sum_{\mathfrak{a}} \lambda_{P}(\mathfrak{a}) q^{N(\mathfrak{a})}$, where $\mathfrak{a}$ runs over all integral ideals of $M$. By $k(P) \geq 2$ (and (Gal)), $M$ has to be an imaginary quadratic field in which $p$ is split (as the holomorphic binary theta series of real quadratic fields are limited to weight 1 ; cf. [MFM, $\S 4.8])$. This shows that $(\mathrm{CM} 1) \Rightarrow(\mathrm{CM} 2) \Rightarrow(\mathrm{CM} 3)$. If (CM2) is satisfied, we have an identity

$$
\operatorname{Tr}\left(\rho_{\mathbb{I}}\left(F r o b_{l}\right)\right)=a(l)=\chi(l) a(l)=\operatorname{Tr}\left(\rho_{\mathbb{I}} \otimes \chi\left(F r o b_{l}\right)\right)
$$

with $\chi=\left(\frac{M / \mathbb{Q}}{)}\right)$ for all primes $l$ outside $N p$. By Chebotarev density, we have $\operatorname{Tr}\left(\rho_{\mathbb{I}}\right)=\operatorname{Tr}\left(\rho_{\mathbb{I}} \otimes \chi\right)$, and we get (CM1) from (CM2) as $\rho_{\mathbb{I}}$ is semi-simple. If a component $\operatorname{Spec}(\mathbb{I})$ contains an arithmetic point $P$ with theta series $f_{P}$ as above of $M / \mathbb{Q}$, either $\mathbb{I}$ is a $\mathrm{CM}$ component or otherwise $P$ is in the intersection in $\operatorname{Spec}(\mathbf{h})$ of a component $\operatorname{Spec}(\mathbb{I})$ not having CM by $M$ and another component having CM by $M$ (as all families with CM by $M$ are made up of theta series of $M$ by the construction of CM components in [H86a, $\S 7]$ ). The latter case cannot happen as two distinct components never cross at an arithmetic point in $\operatorname{Spec}(\mathbf{h})$ (i.e., the reduced part of the localization $\mathbf{h}_{P}$ is étale over $\Lambda_{P}$ for any arithmetic point $P \in \operatorname{Spec}(\Lambda)\left(\overline{\mathbb{Q}}_{p}\right)$; see [HMI Proposition 3.78]). Thus (CM3) implies (CM2). We call a binary theta series of the norm form of an imaginary quadratic field a $C M$ theta series.

\section{WEIL NUMBERS}

Since $\overline{\mathbb{Q}}$ sits inside $\mathbb{C}$, it has "the" complex conjugation $c$. For a prime $l$, a Weil $l$-number $\alpha \in \overline{\mathbb{Q}}$ of integer weight $k \geq 0$ is defined by the following two properties:

(1) $\alpha$ is an algebraic integer;

(2) $\left|\alpha^{\sigma}\right|=l^{k / 2}$ for all $\sigma \in \operatorname{Gal}(\overline{\mathbb{Q}} / \mathbb{Q})$ for the complex Archimedean absolute value $|\cdot|$.

Note that $\mathbb{Q}(\alpha)$ is in a CM field finite over $\mathbb{Q}$ (e.g., [Ho, Proposition 4]).

Proposition 2.1. Let $\alpha$ be a Weil p-number of weight $k$. Write $R$ (resp. $R^{+}$) for the integer ring of $\mathbb{Q}(\alpha)$ (resp. of the maximal real subfield of $\mathbb{Q}(\alpha)$ ). Suppose that one of the following two conditions holds:

(1) $k=1$ and the prime $p$ is unramified in $\mathbb{Q}(\alpha) / \mathbb{Q}$, 
(2) $k \geq 1$ and $\alpha$ generates over $R_{p}^{+}:=R^{+} \otimes_{\mathbb{Z}} \mathbb{Z}_{p}$ the integral closure of $\mathbb{Z}_{p}$ in $R \otimes_{\mathbb{Z}} \mathbb{Q}_{p}$

If we have $|\alpha|_{p}=\left|i_{p}(\alpha)\right|_{p}=1$ for the embedding $i_{p}: \overline{\mathbb{Q}} \hookrightarrow \mathbb{C}_{p}$ (fixed in the introduction), then the set of all p-adic places of $\mathbb{Q}(\alpha)$ splits into a disjoint union $\Sigma_{p} \sqcup \Sigma_{p}^{c}$ for the complex conjugation $c$ such that $\mathfrak{P} \mid(\alpha) \Leftrightarrow \mathfrak{P} \in \Sigma_{p}^{c}$; indeed, $(\alpha)=\prod_{\mathfrak{P} \in \Sigma_{p}^{c}} \mathfrak{P}^{e(\mathfrak{P}) k}$ for the ramification index $e(\mathfrak{P})$ of $\mathfrak{P} / p$.

Proof. Write $K=\mathbb{Q}(\alpha)$, and take its Galois closure $K^{\text {gal }}$ over $\mathbb{Q}$ inside $\overline{\mathbb{Q}}$. Since $K$ is a CM field, the complex conjugation $c$ is in the center $Z$ of $G=\operatorname{Gal}\left(K^{\text {gal }} / \mathbb{Q}\right.$ ) (see IAT, Proposition 5.11]). Since decomposition groups of a prime in $G$ are conjugate to each other, $c$ is either inside all of them or outside all of them. Let $\Sigma:=\Sigma_{\alpha}=$ $\left\{\sigma:\left.K \hookrightarrow \mathbb{C}_{p}|| \alpha^{\sigma}\right|_{p}=1\right\}$ and write $\Sigma_{p}$ for the set of prime ideals $\mathfrak{P}$ of $K$ such that $\mathfrak{P}^{c}=\left\{\left.\xi \in K|| \sigma(\xi)\right|_{p}<1\right\}$. By assumption, $\Sigma \neq \emptyset$; so, no $p$-decomposition groups in $G$ contain $c$. Therefore, any prime factor of $p$ in the maximal totally real field $K_{+}$splits completely in $K$. Write the prime decomposition of the principal ideal $(\alpha)$ as $(\alpha)=\prod_{\mathfrak{P} \mid p} \mathfrak{P}^{\varepsilon(\mathfrak{P})}$. Since $\sigma(\alpha) c \sigma(\alpha)=p^{k}$ implies $\varepsilon(\mathfrak{P})+\varepsilon\left(\mathfrak{P}^{c}\right)=k$, under the assumption (1), unramifiedness of $p$ tells us that $0 \leq \varepsilon(\mathfrak{P}), \varepsilon\left(\mathfrak{P}^{c}\right) \leq 1$, and hence one of them vanishes. Thus $\{\mathfrak{P} \mid p\}=\Sigma_{p} \sqcup \Sigma_{p}^{c}$, and $(\alpha)=\prod_{\mathfrak{P} \in \Sigma_{p}^{c}} \mathfrak{P}$.

Now assume $(2)$. Decompose $(\alpha)=\prod_{\mathfrak{P} \mid p} \mathfrak{P}^{\varepsilon(\mathfrak{P})}$. Write $R_{\mathfrak{P}}$ for the $\mathfrak{P}$-adic completion of $R$. Then for $\mathfrak{p}=\mathfrak{P} \cap R_{+}, R_{\mathfrak{p}}=R_{\mathfrak{P}} \times R_{\mathfrak{P}^{c}}$. If $\varepsilon(\mathfrak{P})>0$ and $\varepsilon\left(\mathfrak{P}^{c}\right)>0$, $\alpha \equiv c(\alpha) \bmod \mathfrak{p} R_{\mathfrak{p}}$ inside $R_{\mathfrak{p}}$, which implies that $R_{p}^{+}[\alpha]$ in $R_{p}$ is contained in the order of conductor $\mathfrak{p}$; so, $\alpha$ cannot span the integral closure $R_{p}$ of $\mathbb{Z}_{p}$ in $\mathbb{Q}(\alpha) \otimes_{\mathbb{Z}} \mathbb{Z}_{p}$. In other words, under $(2), \varepsilon(\mathfrak{P}) \varepsilon\left(\mathfrak{P}^{c}\right)=0$, which implies that $\{\mathfrak{P} \mid p\}=\Sigma_{p} \sqcup \Sigma_{p}^{c}$ and $(\alpha)=\prod_{\mathfrak{P} \in \Sigma_{p}^{c}} \mathfrak{P}^{e(\mathfrak{P}) k}$, as desired.

Corollary 2.2. Let the notation and assumption be as in the above proposition. Let $V$ be a discrete valuation ring finite flat over $\mathbb{Z}_{p}$ with residue field $\mathbb{F}_{p}$. Let $M$ be a CM field containing $\mathbb{Q}(\alpha)$, and let $A_{/ V}$ be a polarized abelian scheme of dimension $[M: \mathbb{Q}]$ with a ring embedding $\theta: M \hookrightarrow \operatorname{End}\left(A_{/ V}\right) \otimes_{\mathbb{Z}} \mathbb{Q}$ sending the identity to the identity whose image is stable under the Rosati involution. Put $\widetilde{A}=A \times_{V} \mathbb{F}_{p}$ (the reduction modulo $p)$. If $F^{k}-\theta(\alpha) \in \operatorname{End}\left(\widetilde{A}_{\mathbb{F}_{p}}\right)$ has kernel of positive dimension for the relative p-power Frobenius endomorphism $F$ of $A \times_{V} \mathbb{F}_{p}$, then $\widetilde{A}$ is an ordinary abelian variety.

Proof. Take a prime $l \neq p$. The $l$-adic Tate module $T_{l} \widetilde{A} \otimes_{\mathbb{Z}_{l}} \mathbb{Q}_{l}$ is free of rank 2 over $M_{l}=M \otimes_{\mathbb{Q}} \mathbb{Q}_{l}$, and $H\left(F^{k}\right)=0$ for the polynomial $H(T):=T^{2}-a T+p=0$ $\left(a=\theta\left(\alpha+\alpha^{c}\right)\right)$ with coefficients in the maximal real subfield of $M$, as the Rosati involution coincides with complex conjugation $c$ on $M$. Replacing $A$ by an isogenous abelian scheme, we may assume that $\theta^{-1}\left(\operatorname{End}\left(A_{/ V}\right)\right)$ contains the integer ring $\mathfrak{O}$ of $M$ (e.g., [IAT, (7.7.8)]). Thus by the above proposition, $a \bmod p$ is a unit in $\mathfrak{O} / p \mathfrak{O}$ and hence $\widetilde{A}$ is an ordinary abelian variety.

We call two nonzero numbers $a, b \in \overline{\mathbb{Q}}$ equivalent (written as $a \sim b$ ) if $a / b$ is a root of unity.

Lemma 2.3. Let $K$ be a finite field extension of $\mathbb{Q}\left(\mu_{p^{\infty}}\right)$ inside $\overline{\mathbb{Q}}$. Then for a given prime $l$ and weight $k \geq 0$, there are only finitely many Weil $l$-numbers of weight $k$ in $K$ up to equivalence. If $K=\mathbb{Q}\left(\mu_{p^{\infty}}\right)$ and there is only one prime in $\mathbb{Z}\left[\mu_{p^{\infty}}\right]$ above $(l)$ (for example, if $l=p$ ), any Weil l-number of weight $k$ is equivalent 
to $\left(l^{*}\right)^{k / 2}$ (as long as $\left(l^{*}\right)^{k / 2}$ is in $K$ ), where $l^{*}=(-1)^{(l-1) / 2} l$ if $l$ is odd, and $l^{*}=2$ if $l=2$.

A result of Loxton confirms that, up to equivalence, there are only finitely many Weil $l$-numbers of a given weight in the maximal abelian extension $\mathbb{Q}^{a b}$ of $\mathbb{Q}$ (see Lo, Lemma 7]). Thus the finiteness result of the lemma follows from this analytic result of Loxton. We will give an algebraic proof.

Proof. The decomposition group of each prime $l$ is of finite index in $\operatorname{Gal}\left(\mathbb{Q}\left[\mu_{p^{\infty}}\right] / \mathbb{Q}\right)$ (identifying $\operatorname{Gal}\left(\mathbb{Q}\left[\mu_{p} \infty\right] / \mathbb{Q}\right) \cong \mathbb{Z}_{p}^{\times}$by the $p$-adic cyclotomic character, the decomposition group is generated by $l$ if $l \neq p$, and otherwise if $l=p$, then $p$ is fully ramified in $\mathbb{Q}\left[\mu_{p^{\infty}}\right]$; see $[\mathrm{ICF}$, Chapter 2$]$ ); so, there are only finitely many primes $\mathfrak{L}$ of $\mathbb{Z}\left[\mu_{p^{\infty}}\right]$ above $(l)$. Thus for a Weil $l$-number $\alpha$ of weight $k$, there are only finitely many possibilities of prime factorization of $(\alpha)$ if $l \neq p$. If $(\alpha)=(\beta)$ for two Weil $l$-numbers $\alpha, \beta$, then $\alpha / \beta$ is a Weil number of weight 0 ; so, $\alpha \sim \beta$ by Kronecker's theorem. If there is only one prime over $l$ in $\mathbb{Z}\left[\mu_{p^{\infty}}\right]$, any Weil $l$-number of weight $k$ is equivalent to $\left(l^{*}\right)^{k / 2}$, as long as $\left(l^{*}\right)^{k / 2} \in \mathbb{Q}\left[\mu_{p^{\infty}}\right]$. Thus the result follows from this if $K=\mathbb{Q}\left(\mu_{p} \infty\right)$.

Let $W(l, k)$ (resp. $\left.W_{K}(l, k)\right)$ be a complete set of representatives of Weil $l$ numbers in $\mathbb{Q}\left(\mu_{p} \infty\right)$ (resp. in $K$ ) of weight $k$ under the equivalence. By the above argument, $W(l, k)$ is a finite set, and we want to prove that $W_{K}(l, k)$ is finite. Write $d=\left[K: \mathbb{Q}\left(\mu_{p^{\infty}}\right)\right]$. If $\alpha \in K$ is such a Weil $l$-number, then $N_{K / \mathbb{Q}\left(\mu_{p} \infty\right)}(\alpha)$ is equivalent to a number in $W(l, k d)$. Thus $N_{K / \mathbb{Q}\left(\mu_{p} \infty\right)}$ induces a map $N: W_{K}(l, k) \rightarrow$ $W(l, d k)$. Write $L$ for the field generated by elements in $W(l, d k)$. Then $L / \mathbb{Q}$ is a finite abelian extension in $\mathbb{Q}\left(\mu_{p^{\infty}}\right)$. Since no prime completely splits in $\mathbb{Q}\left(\mu_{p^{\infty}}\right)$, the decomposition subgroup $D$ of $l$ in $\operatorname{Gal}(K / \mathbb{Q})$ is an open subgroup of finite index. Thus there are only finitely many valuations $v$ of $K$ with $v(l)=1$. Let $\mathcal{V}$ be the set of valuations $v$ of $K$ with $v(l)=1$, which is a finite set. For $v \in \mathcal{V}$ and $\alpha \in W_{K}(l, k), v(\alpha) \in[0, k] \cap d^{-1} v(L)$, because $N_{K / \mathbb{Q}\left(\mu_{p} \infty\right)}(\alpha)$ is in $W(l, d k)$ up to roots of unity. Let $V:=\prod_{v \in \mathcal{V}}\left([0, k] \cap d^{-1} v(L)\right)$, which is a finite set. We have a $\operatorname{map}_{\operatorname{ord}}: W_{K}(l, k) \rightarrow V$ sending $\alpha$ to $\operatorname{ord}_{l}(\alpha)=(v(\alpha))_{v \in \mathcal{V}}$. If $\operatorname{ord}_{l}(\alpha)=\operatorname{ord}_{l}(\beta)$ $\left(\alpha, \beta \in W_{K}(l, k)\right)$, then $\alpha / \beta$ is an algebraic integer with complex absolute value $\left|(\alpha / \beta)^{\sigma}\right|=1$ for all $\sigma \in \operatorname{Gal}(\overline{\mathbb{Q}} / \mathbb{Q})$; so, by Kronecker's theorem, $\alpha \sim \beta$. Thus ord is an injection, proving the finiteness of $W_{K}(l, k)$.

Proposition 2.4. Let $G$ be a finite group and $l$ be a prime. Let $\mathcal{K}_{G}$ be the set of all Galois extensions of $K:=\mathbb{Q}\left[\mu_{p} \infty\right]$ with Galois group $G$ inside $\overline{\mathbb{Q}}$ whose ramification at $l$ over $K$ is tame. Then there are only finitely many Weil l-numbers of a given weight in the set-theoretic union $\bigcup_{L \in \mathcal{K}_{G}} L($ in $\overline{\mathbb{Q}})$ up to equivalence.

The point of the proof is as follows (and we fill in the details after the outline). Write $K_{l}=K \otimes_{\mathbb{Q}} \mathbb{Q}_{l}$. By tameness, there are only finitely many isomorphism classes of $K_{l}$-algebras $L_{l}=L \otimes_{\mathbb{Q}} \mathbb{Q}_{l}$ for $L \in \mathcal{K}_{G}$. Thus we only need to prove finiteness for Weil numbers of a given weight contained in a fixed isomorphism class of $L_{l}$. We look at the universal composite $L_{l} \otimes_{K_{l}} L_{l}$, which is a product of fields indexed by $l$-adic nonequivalent valuations $\mathcal{V}_{1}, \ldots, \mathcal{V}_{n}$ normalized so that $\mathcal{V}_{i}(l)=1$. Consider a tuple

$$
\nu(\alpha)=\left(\mathcal{V}_{1}(\alpha \otimes 1), \ldots, \mathcal{V}_{n}(\alpha \otimes 1), \mathcal{V}_{1}(1 \otimes \alpha), \ldots, \mathcal{V}_{n}(1 \otimes \alpha)\right)
$$

If $\alpha \sim \beta$, we have $\nu(\alpha)=\nu(\beta)$. The tuple $\nu(\alpha)$ determines the prime factorization of $(\alpha)$ in any possible composite $K(\alpha, \beta)$; so, if $\nu(\alpha)=\nu(\beta),(\alpha)=(\beta)$ in $K(\alpha, \beta)$; 
so, by Kronecker's theorem, $\alpha \sim \beta$. Since there are only finitely many possibilities of $\nu(\alpha)$, there are only finitely many classes.

Proof. Let $\mathcal{V}$ be the set of normalized $l$-adic valuations of $K:=\mathbb{Q}\left[\mu_{p^{\infty}}\right]$. Write $\mathbb{F}_{v}$ for the residue field of $v \in \mathcal{V}$ inside a fixed algebraic closure $\overline{\mathbb{F}}_{l}$. If $l \neq p$, then $\mathbb{F}_{v}$ is an infinite extension of $\mathbb{F}_{l}$, and otherwise, $\mathbb{F}_{v}=\mathbb{F}_{p}$. As we have remarked, $\mathcal{V}$ is a finite set. Let $L \in \mathcal{K}_{G}$; so, $L / K$ is a finite extension of degree $d=|G|$ tamely ramified at $l$. We claim that there are only finitely many isomorphism classes of $K_{l}$-algebras $L_{l}=L \otimes_{\mathbb{Q}} \mathbb{Q}_{l}$ for $L$ running through $\mathcal{K}_{G}$. To see this, write $K_{l}=K \otimes_{\mathbb{Q}} \mathbb{Q}_{l}=\prod_{v \in \mathcal{V}} K_{v}$ for $K_{v}=\mathbb{Q}_{l}\left[\mu_{p^{\infty}}\right]$ inside the $v$-adic completion of $K$. Let $K_{v}^{t} / K_{v}$ (resp. $K_{v}^{u r} / K_{v}$ ) be the maximal tamely $l$-ramified extension (resp. the maximal unramified extension inside $K_{v}^{t}$ ). Then by class field theory, $K_{v}^{t}$ has the $l$-inertia group isomorphic to $\widehat{\mathbb{Z}}^{(l)}(1)$, where $\widehat{\mathbb{Z}}^{(l)}(1)=\varliminf_{l \nmid N} \mu_{N}\left(\overline{\mathbb{Q}}_{l}\right)$ as $\operatorname{Gal}\left(K_{v}^{u r} / \mathbb{Q}_{p}\right)$-modules. Thus we have

$$
\operatorname{Gal}\left(K_{v}^{t} / K_{v}\right) \cong \begin{cases}\widehat{\mathbb{Z}}^{(p)} \ltimes \widehat{\mathbb{Z}}^{(l)}(1) & \text { if } l \neq p, \\ \widehat{\mathbb{Z}} \ltimes \widehat{\mathbb{Z}}^{(l)}(1) & \text { if } l=p\end{cases}
$$

(a semi-direct product with $\left.\operatorname{Gal}\left(K_{v}^{t} / K_{v}\right) \triangleright \widehat{\mathbb{Z}}^{(l)}(1)\right)$, since

$$
\operatorname{Gal}\left(K_{v}^{u r} / K_{v}\right) \cong \begin{cases}\operatorname{Gal}\left(\overline{\mathbb{F}}_{l} / \mathbb{F}_{v}\right) \cong \widehat{\mathbb{Z}}^{(p)} & \text { if } l \neq p, \\ \widehat{\mathbb{Z}} & \text { if } l=p .\end{cases}
$$

Thus there are finitely many open subgroups of $\operatorname{Gal}\left(K_{v}^{t} / K_{v}\right)$ with index $\leq d$, and hence there are finitely many isomorphism classes of tamely ramified extensions of $K_{v}$ of degree $\leq d$. Thus the claim follows.

Consider the set $\Xi$ of all isomorphism classes of $K_{l}$-algebras $L \otimes_{\mathbb{Q}} \mathbb{Q}_{l}$, where $L$ runs over $\mathcal{K}_{G}$. Thus $\Xi$ is a finite set. Pick $\mathcal{L} \in \Xi$ and consider the set $\mathcal{K}_{\mathcal{L}}$ of all $L \in \mathcal{K}_{G}$ such that $L \otimes_{\mathbb{Q}} \mathbb{Q}_{l} \cong \mathcal{L}$ as $K$-algebras. Then $\mathcal{K}_{G}=\bigsqcup_{\mathcal{L} \in \Xi} \mathcal{K}_{\mathcal{L}}$. The set $\mathcal{K}_{\mathcal{L}}$ is a countable set. Assume $\mathcal{K}_{\mathcal{L}} \neq \emptyset$. We just order its elements as $L_{1}, L_{2}, \ldots$. We fix a $K$-algebra isomorphism $\iota_{j}: L_{j} \otimes_{\mathbb{Q}} \mathbb{Q}_{l} \cong \mathcal{L}$. Thus $L_{j} \subset \mathcal{L}$ for every $j$. We take the (set-theoretic) union $\Omega_{\mathcal{L}}:=\bigcup_{j=1}^{\infty} \iota_{j}\left(L_{j}\right)$ inside $\mathcal{L}$. Since $L_{j} / K$ is a Galois extension, another choice of $\iota_{j}$ is equal to $\iota_{j} \circ \sigma$ for $\sigma \in \operatorname{Gal}\left(L_{j} / K\right)$. Thus $\operatorname{Im}\left(\iota_{j}: L_{j} \rightarrow \mathcal{L}\right)$ is independent of the choice of $\iota_{j}$, and we therefore forget about $\iota_{j}$. Note that $K_{l}=K \otimes_{\mathbb{Q}} \mathbb{Q}_{l}$ is a product of the field $K_{v}$ indexed by $v \in \mathcal{V}$ such that $K_{v}$ has a valuation inducing $v$ on $K$. Similarly $\mathcal{L}$ is a product of finitely many fields with $l$-adic valuation. Write $\mathcal{L}=\prod_{j=1}^{r} \mathbb{L}_{j}$ for a simple component $\mathbb{L}_{j}$, and write $V_{j}$ for the valuation giving the $l$-adic topology of $\mathbb{L}_{j}$ extending one of the members of $\mathcal{V}$. Write $\mathcal{V}_{\mathcal{L}}=\left\{V_{1}, \ldots, V_{r}\right\}$, which is a finite set.

Let $\mathcal{W}(l, k)$ (resp. $\mathcal{W}(l, k, \mathcal{L}))$ be the set of Weil $l$-numbers of weight $k$ in $\bigcup_{L \in \mathcal{K}_{G}} L$ (resp. in $\Omega_{\mathcal{L}}$ ). Thus for each $\alpha \in \mathcal{W}(l, k, \mathcal{L})$, we find a Galois extension $L_{\alpha} / K$ containing $L(\alpha)$ over $K$ inside $\mathcal{K}_{G}$ with Galois group $G$ such that $L_{\alpha} \otimes_{\mathbb{Q}} \mathbb{Q}_{l} \cong \mathcal{L}$. Thus we have

$$
\mathcal{W}(l, k)=\bigcup_{\mathcal{L} \in \Xi} \mathcal{W}(l, k, \mathcal{L})
$$

The intersection $\mathcal{W}(l, k, \mathcal{L}) \cap \mathcal{W}\left(l, k, \mathcal{L}^{\prime}\right)$ for $\mathcal{L}, \mathcal{L}^{\prime} \in \Xi$ obviously contains all Weil $l$-numbers of weight $k$ in $K$ (and possibly more). For each $\alpha \in \mathcal{W}(l, k, \mathcal{L})$, we define a tuple of nonnegative positive rational numbers $S_{1}(\alpha) \in\left(\mathbb{Q}_{\geq 0}\right)^{\mathcal{V}_{\mathcal{L}}}$ given by $S_{1}(\alpha)=(V(\alpha))_{V \in \mathcal{V}_{\mathcal{L}}}$. 
If $l \neq p, l$ is unramified in $K / \mathbb{Q}$; so, $V(\alpha) \in|G|^{-1} \mathbb{Z}$ and $0 \leq V(\alpha) \leq k$. Thus

$$
\mathcal{S}:=\left\{S_{1}(\alpha) \mid \alpha \in \mathcal{W}(l, k, \mathcal{L})\right\}
$$

is a finite set. If $l=p$, by the above lemma, there are only finitely many Weil $p$-numbers (up to equivalence) in $K$ of a given weight. Thus the set $\mathbb{V} \subset \mathbb{Q}$ of valuations of Weil numbers in $K$ of weight $k|G|$ is a finite set. For each $\alpha \in \mathcal{W}(p, k, \mathcal{L})$, take a Galois extension $L_{\alpha} / K$ (inside $\mathcal{K}_{G}$ ) with $\operatorname{Gal}\left(L_{\alpha} / K\right)=G$ containing $\alpha$ with $L_{\alpha} \otimes_{\mathbb{Q}} \mathbb{Q}_{l} \cong \mathcal{L}$. Then $\sum_{V \in \mathcal{V}_{\mathcal{L}}} e_{V} V(\alpha)=v\left(N_{L_{\alpha} / K}(\alpha)\right) \in \mathbb{V}$ for the (tame) ramification index $e_{V}$ of $V$ over $K$ and $V(\alpha) \in|G|^{-1} \mathbb{V}$. Again,

$$
\mathcal{S}:=\left\{S_{1}(\alpha) \mid \alpha \in \mathcal{W}(p, k, \mathcal{L})\right\}
$$

is a finite set. If $\alpha / \beta \in \mu_{p \infty}(K)$, we have $K(\alpha)=K(\beta)$ inside $\mathcal{L}$ and hence $S_{1}(\alpha)=S_{1}(\beta)$. By Lemma 2.6 following this proposition, the group of roots of unity in the composite $\mathbf{L}$ of $L$ for all $L \in \mathcal{K}_{\mathcal{L}}$ in $\overline{\mathbb{Q}}$ contains $\mu_{p^{\infty}}(K)$ as a subgroup of finite index; so, $S_{1}(\alpha)=S_{1}(\beta)$ up to permutation of valuations in $\mathcal{V}_{\mathcal{L}}$ if $\alpha \sim \beta$.

We consider for each $s \in \mathcal{S}$ the set $\mathcal{W}(s)=\left\{\alpha \in \mathcal{W}(l, k, \mathcal{L}) \mid S_{1}(\alpha)=s\right\}$. By definition, we have a decomposition

$$
\mathcal{W}(l, k, \mathcal{L})=\bigsqcup_{s \in \mathcal{S}} \mathcal{W}(s)
$$

We want to show that $\mathcal{W}(s) / \mu_{p^{\infty}}(K)$ is a finite set. Write $\mathcal{O}$ for the integral closure of $\mathbb{Z}_{l}$ in $\mathcal{L}$ (the $l$-adic integer ring of $\mathcal{L}$ ). By definition, for $\alpha, \beta \in \mathcal{W}(s)$, the principal $\mathcal{O}$-ideal $(\alpha)$ is equal to $(\beta)$, because $S_{1}(\alpha)=S_{1}(\beta)=s$ determines the prime factorization of $(\alpha)=(\beta)$ in $\mathcal{L}$. Write $\mathcal{L} \otimes_{K_{l}} \mathcal{L}=\mathcal{L}_{1} \oplus \mathcal{L}_{2} \oplus \cdots \oplus \mathcal{L}_{n}$ for fields $\mathcal{L}_{i}$ and write $\mathcal{V}_{i}$ for the valuation of $\mathcal{L}_{i}$ normalized so that it extends a member of $\mathcal{V}$. Then the possibilities of $\nu(\alpha)=\left(\mathcal{V}_{i}\left((\alpha \otimes 1), \mathcal{V}_{i}(1 \otimes \alpha)\right)_{i}\right.$ for $\alpha$ running over $\mathcal{W}(s)$ are finitely many, and if $\nu(\alpha)=\nu(\beta), \alpha / \beta$ is a Weil $l$-number of weight 0 , because the prime factorizations of $(\alpha)$ and $(\beta)$ in $L_{\alpha} \otimes_{K} L_{\beta}$ are equal. Thus $\alpha / \beta$ is a root of unity by Kronecker's theorem, and hence $\alpha \sim \beta$. As already remarked, $\{$ roots of unity in $\mathbf{L}\}=\bigcup_{\zeta \in Z} \zeta \cdot \mu_{p^{\infty}}(K)$ for a finite set $Z$ of roots of unity. Thus $\mathcal{W}(s) / \mu_{p^{\infty}}(K)$ is finite, which implies that $\mathcal{W}(l, k) /\{$ roots of unity $\}$ is finite.

Corollary 2.5. Let $d$ be a positive integer. Let $\mathcal{K}_{d}$ be the set of all finite extensions of $\mathbb{Q}\left[\mu_{p^{\infty}}\right]$ of degree $d$ inside $\overline{\mathbb{Q}}$ whose ramification at l is tame. Then there are only finitely many Weil l-numbers of a given weight in the set-theoretic union $\bigcup_{L \in \mathcal{K}_{d}} L$ (in $\overline{\mathbb{Q}})$ up to equivalence.

Proof. The Galois closure $L^{\text {gal }}$ of $L \in \mathcal{K}_{d}$ over $K=\mathbb{Q}\left[\mu_{p^{\infty}}\right]$ has Galois group which is isomorphic to a subgroup of the permutation group $\mathfrak{S}_{d}$ of $d$ letters. Thus the possible isomorphism classes of $\operatorname{Gal}\left(L^{g a l} / K\right)$ are finite. Note that $l$ tamely ramifies in $L^{g a l} / K$. Thus applying the above proposition for each $G \subset \mathfrak{S}_{d}$, we get the desired result.

Lemma 2.6. Let $\mathcal{K}_{\text {? }}$ be one of $\mathcal{K}_{\mathcal{L}}, \mathcal{K}_{G}$ and $\mathcal{K}_{d}$. Suppose $\mathcal{K}_{\text {? }} \neq \emptyset$. Then the group of roots of unity in the composite $\mathbf{L}$ of $L$ for $L \in \mathcal{K}_{\text {? }}$ in $\overline{\mathbb{Q}}$ contains $\mu_{p^{\infty}}(K)$ as a subgroup of finite index.

Proof. We first take $\mathcal{K}_{?}=\mathcal{K}_{\mathcal{L}}$ or $\mathcal{K}_{G}$. Let $\mu^{(p)} \subset \overline{\mathbb{Q}}$ be the group of roots of unity of order prime to $p$. We need to prove that the subgroup $\mu^{(p)} \cap \mathbf{L} \subset \mu^{(p)}$ is finite. 
For any finite subset $\mathcal{K} \subset \mathcal{K}_{\text {? }}$, the composite of $L \in \mathcal{K}$ in $\overline{\mathbb{Q}}$ is a Galois extension of $K$ whose Galois group is a subgroup of $\prod_{L \in \mathcal{K}} G$ contained in

$$
\left\{\left(\sigma_{L}\right)_{L \in \mathcal{K}}\left|\sigma_{L} \in \operatorname{Gal}(L / K), \sigma_{L}\right|_{L \cap L^{\prime}}=\left.\sigma_{L^{\prime}}\right|_{L \cap L^{\prime}} \text { for all pairs }\left(L, L^{\prime}\right) \in \mathcal{K}^{2}\right\} .
$$

Thus any $\sigma \in \operatorname{Gal}(\mathbf{L} / K)$ has order a factor of $|G|$, so is bounded. Pick any infinite subgroup $\nu \subset \mu^{(p)}$, and decompose it into a product of $\ell$-primary subgroups $\nu_{\ell}$ for primes $\ell \neq p$. Let $\Xi=\left\{\ell \mid \nu_{\ell} \neq 1\right\}$. If $\Xi$ is finite, we have $\nu \supset \mu_{\ell \infty}$ for a prime $\ell \in \Xi$, and $\operatorname{Gal}(K(\nu) / K)$ surjects down to $\operatorname{Gal}\left(\mathbb{Q}\left(\mu_{\ell}\right) / \mathbb{Q}\right)=\mathbb{Z}_{\ell}^{\times}$; so, the elements of $\operatorname{Gal}(K(\nu) / K)$ have unbounded order, and hence $\mathbb{Q}(\nu) \not \subset \mathbf{L}$. If $|\Xi|=\infty$, $\operatorname{Gal}\left(K\left(\nu_{\ell}\right) / K\right)$ for odd $\ell$ is cyclic of order $\geq \ell-1$, and therefore, elements of $\operatorname{Gal}(K(\nu) / K)$ have unbounded order. Thus the subgroup $\mu^{(p)} \cap \mathbf{L}$ has to be finite. As for $\mathcal{K}_{?}=\mathcal{K}_{d}$, the Galois closure of $L \in \mathcal{K}_{d}$ over $K$ has Galois group $G$ realized inside $\mathfrak{S}_{d}$, and the same proof works since what we used is only the boundedness of $|G| \leq d !$.

\section{Theorems AND CONJECTURES}

Let $f=\sum_{n=1}^{\infty} a(n, f) q^{n} \in S_{k}\left(\Gamma_{0}\left(N p^{r} \mathbf{p}\right), \psi\right)$ be a Hecke eigenform normalized so that $f \mid T(n)=a(n, f) f$ for all $n$. We write $f \mid T(l)=\left(\alpha_{l}+\beta_{l}\right) f$ and $\alpha_{l} \beta_{l}=\psi(l) l^{k-1}$ if $l \nmid N p^{r+1}\left(\alpha_{l}, \beta_{l} \in \overline{\mathbb{Q}}\right)$. If $l \mid N p^{r+1}$, we put $\beta_{l}=0$ and define $\alpha_{l} \in \overline{\mathbb{Q}}$ by $f \mid U(l)=\alpha_{l} f$. Then the Hecke polynomial $H_{l}(X)=\left(1-\alpha_{l} X\right)\left(1-\beta_{l} X\right)$ gives the Euler $l$-factor of $L(s, f)=\sum_{n=1}^{\infty} a(n, f) n^{-s}$ after replacing $X$ by $l^{-s}$ and inverting the factor.

Let $\mathcal{F}=\left\{f_{P}\right\}_{P \in \operatorname{Spec}(\mathbb{I})\left(\mathbb{C}_{p}\right)}$ be a $p$-adic analytic family of $p$-ordinary Hecke eigen cusp forms of slope 0 . We write $\alpha_{l, P}, \beta_{l, P}$ for $\alpha_{l}, \beta_{l}$ for $f_{P}$. We have the following two versions of the conjecture:

Conjecture 3.1 (Vertical version).

(Weak form): The field $\mathbb{Q}_{V, r}(\mathcal{F})$ is a finite extension of $\mathbb{Q}$ for a fixed $r<\infty$ if and only if $f_{P}$ is a CM theta series for some arithmetic $P$ with $k(P) \geq 2$.

(Strong form): Let $\mathcal{A}$ be an infinite set of arithmetic points with bounded level $r(P) \leq r$ for a fixed $r \geq 0$ (so, $\operatorname{Im}\left(\varepsilon_{P}\right) \subset \mu_{p^{r}}$ for the fixed $\left.r\right)$. Let $M_{V, \mathcal{A}}(\mathcal{F})$ be the field generated over $\mathbb{Q}$ by $\left\{\alpha_{p, P}\right\}_{P \in \mathcal{A}}$, where $P$ runs over all points in $\mathcal{A}$. Then the field $M_{V, \mathcal{A}}(\mathcal{F})$ is a finite extension of $\mathbb{Q}$ for a fixed $r<\infty$ if and only if $f_{P}$ is a $C M$ theta series for some arithmetic $P$ with $k(P) \geq 2$.

Pick a prime $l$ different from $p$ and write $M_{V, \mathcal{A}}^{(l)}(\mathcal{F})$ for the field generated by $\left\{\alpha_{l, P}, \beta_{l, P}\right\}_{P \in \mathcal{A}}$, where $P$ runs over all points in $\mathcal{A}$. Then we might speculate that

Vertical $l$-version of strong form. The field $M_{V, \mathcal{A}}^{(l)}(\mathcal{F})$ is a finite extension of $\mathbb{Q}$ for a fixed $r<\infty$ if and only if for an arithmetic $P$ with $k(P) \geq 2$, either $f_{P}$ is a $C M$ theta series or the automorphic representation generated by $f_{P}$ is squareintegrable at $l$.

What we can prove is as follows.

Theorem 3.2 (Strong vertical theorem). Let $r$ be a nonnegative integer. Assume $p>2$ and that there exists an arithmetic point $P_{0} \in \operatorname{Spec}(\mathbb{I})\left(\mathbb{C}_{p}\right)$ with $k\left(P_{0}\right) \geq 2$ such that $\alpha_{0}=a\left(p, f_{P_{0}}\right)$ is a Weil number and $\Sigma_{\alpha_{0}}=\left\{\sigma: \mathbb{Q}\left(\alpha_{0}\right) \hookrightarrow \overline{\mathbb{Q}}|| i_{p}\left(\alpha_{0}^{\sigma}\right) \mid=1\right\}$ is a CM type of $\mathbb{Q}\left(\alpha_{0}\right)$. Pick an infinite set $\mathcal{A}$ of arithmetic points $P$ with bounded level $r(P) \leq r$. Then the field $M_{V, \mathcal{A}}(\mathcal{F})$ is a finite extension of $\mathbb{Q}$ if and only if $f_{P}$ is a $C M$ theta series for an arithmetic $P$ with $k(P) \geq 2$. 
See Proposition 2.1 for sufficient conditions which guarantee the truth of the assumption of the theorem. Take the rank 2 motive $M_{0}$ with coefficients in $\mathbb{Q}\left(f_{P_{0}}\right)$ (in $[\mathrm{S}]$ ). Regarding $M_{0}$ as having coefficients in $\mathbb{Q}, M_{0}$ is potentially crystalline at $p$ and ordinary if and only if $\Sigma_{\alpha_{0}}$ is a CM type of $\mathbb{Q}\left(\alpha_{0}\right)$. Here a motive is ordinary if the Newton polygon of Frobenius at $p$ (in our case, the Newton polygon of $\alpha_{0}$ ) coincides with the Hodge polygon. We prove this theorem and the vertical theorem (in the introduction) in Section 8 . The horizontal theorem in the introduction follows from the following version (and Corollary 6.3):

Theorem 3.3 (Strong horizontal theorem). Pick an infinite set $\mathcal{A}$ of arithmetic points $P$ with fixed $k(P)=k \geq 2$. Write $M_{H, \mathcal{A}}(\mathcal{F}) \subset \overline{\mathbb{Q}}$ for the field generated over $\mathbb{Q}\left(\mu_{p^{\infty}}\right)$ by $\left\{\alpha_{p, P}\right\}_{P \in \mathcal{A}}$, where $P$ runs over all arithmetic points in $\mathcal{A}$. Suppose $p>2$. Then the field $M_{H, \mathcal{A}}(\mathcal{F})$ is a finite extension of $\mathbb{Q}\left(\mu_{p^{\infty}}\right)$ if and only if $f_{P}$ is a CM theta series for an arithmetic $P$ with $k(P) \geq 2$.

We prove this theorem in Section 6. For a prime $l \neq p$, we may conjecture the $l$-version of the stronger form in the horizontal case also:

Conjecture 3.4 (Horizontal l-version). Write $M_{H, \mathcal{A}}^{(l)}(\mathcal{F})$ for the field generated over $\mathbb{Q}\left(\mu_{p^{\infty}}\right)$ by $\left\{\alpha_{l, P}\right\}_{P \in \mathcal{A}}$. Then $M_{H, \mathcal{A}}^{(l)}(\mathcal{F})$ is a finite extension of $\mathbb{Q}\left(\mu_{p^{\infty}}\right)$ if and only if for an arithmetic $P$ with $k(P) \geq 2$, either $f_{P}$ is a CM theta series or the automorphic representation generated by $f_{P}$ is square-integrable at $l$.

One can show that $\left[M_{H, \mathcal{A}}^{(l)}(\mathcal{F}): \mathbb{Q}\left(\mu_{p^{\infty}}\right)\right]<\infty$ if $f_{P_{0}}$ is square-integrable at a prime $l \neq p$ for one arithmetic $P_{0}$ as follows: Let $\operatorname{Spec}(\mathbb{I})$ be the irreducible component associated to the family $\mathcal{F}$. If $f_{P_{0}}$ (or, more precisely, the automorphic representation generated by $f_{P_{0}}$ ) is super-cuspidal at $l$, then $\left.\rho_{P_{0}}\right|_{I_{l}}$ is absolutely irreducible (by the local Langlands conjecture for $G L(2)$ solved by Kutzko and Carayol; e.g., $\mathrm{Ca}$ ). Then the Galois representation $\rho_{\mathbb{I}}$ is absolutely irreducible over the inertia group $I_{l}$ and $\rho_{\mathbb{I}}\left(I_{l}\right)$ is finite by (2.1). Therefore, it is rigid; i.e., $\left.\rho_{P}\right|_{I_{l}}$ is independent of points $P \in \operatorname{Spec}(\mathbb{I})$ with characteristic 0 residue field. Thus if $f_{P_{0}}$ is super-cuspidal for one arithmetic point $P_{0}$, then $\left.\rho_{P_{0}}\right|_{I_{l}}$ is irreducible. Therefore, $\left.\rho_{\mathbb{I}}\right|_{I_{l}}$ is irreducible, which implies that $\left.\rho_{P}\right|_{I_{l}}$ is irreducible for all $P$ with characteristic 0 residue field. Hence $f_{P}$ is super-cuspidal for all arithmetic $P$. Then $\alpha_{l}=a(l)=0$ in $\mathbb{I}$; so, $M_{H, \mathcal{A}}^{(l)}(\mathcal{F})=\mathbb{Q}\left(\mu_{p^{\infty}}\right)$ in this case. Suppose that the automorphic representation $\pi_{P_{0}}$ generated by $f_{P_{0}}$ has its local factor at $l$ isomorphic to the Steinberg representation $\sigma\left(\alpha|\cdot|_{l}, \alpha\right)$ for a character $\alpha: \mathbb{Q}_{l}^{\times} \rightarrow \mathbb{C}^{\times}$. If $\alpha$ is unramified, $f_{P_{0}}$ is $l$-new of $l$-conductor $l$, and hence $f_{P}$ is Steinberg for all arithmetic $P$ because the outside $p$-conductor and the outside $p$ central character are constant in the family (the old/new theory at the level of families, e.g., [H88, Theorem 3.6]). If $\alpha$ is ramified, taking a global Dirichlet character $\chi: \mathbb{A}^{\times} / \mathbb{Q}^{\times} \rightarrow \mathbb{C}^{\times}$ with $\left.\chi\right|_{\mathbb{Z}_{l}^{\times}}=\left.\alpha^{-1}\right|_{\mathbb{Z}_{l}^{\times}}$only ramified at $l,\left\{\pi_{P} \otimes \chi\right\}_{P}$ is associated to another analytic family $\mathcal{F} \otimes \chi$ of slope 0 . Replacing $\mathcal{F}$ by $\mathcal{F} \otimes \chi$, we may assume $\alpha$ is unramified; so, if one $f_{P_{0}}$ is Steinberg at $l \neq p$, all members of $\mathcal{F}$ are Steinberg at $l \neq p$. Using this fact, we prove in Proposition 5.2 that $M_{H, \mathcal{A}}^{(l)}(\mathcal{F})$ is a finite extension of $\mathbb{Q}\left(\mu_{p^{\infty}}\right)$ if $f_{P_{0}}$ is Steinberg at $l \neq p$ at one arithmetic $P_{0}$. A similar argument works also for the vertical $l$-version, proving $\left[M_{V, \mathcal{A}}^{(l)}(\mathcal{F}): \mathbb{Q}\right]<\infty$ if $f_{P_{0}}$ is square-integrable at $l \neq p$ for one arithmetic $P_{0}$. 
We will prove the following weaker statements in the following section:

Proposition 3.5. (WV) Let $\widetilde{M}_{V, r}(\mathcal{F})$ be the field generated over $\mathbb{Q}$ by $\left\{\alpha_{l, P}^{2}\right.$, $\left.\beta_{l, P}^{2}\right\}_{l, P}$, where $P$ runs over arithmetic points with $\operatorname{Im}\left(\varepsilon_{P}\right) \subset \mu_{p^{r}}$ for a fixed $r>0$ and $l$ runs over all primes. Then $\widetilde{M}_{V, r}(\mathcal{F})$ is a finite extension of $\mathbb{Q}$ for a fixed $r<\infty$ if and only if $f_{P}$ is a CM theta series for some arithmetic $P$ with $k(P) \geq 2$.

$(\mathrm{WH})$ Let $\widetilde{M}_{H, k}(\mathcal{F})$ be the field generated over $\mathbb{Q}$ by $\left\{\alpha_{l, P}^{2}, \beta_{l, P}^{2}\right\}_{l, P}$, where $P$ runs over all arithmetic points with $k(P)=k$ for a fixed $k \geq 2$ and $l$ runs over all primes. The field $\widetilde{M}_{H, k}(\mathcal{F})$ is a finite extension of $\mathbb{Q}\left(\mu_{p \infty}\right)$ for a fixed $k$ if and only if $f_{P}$ is a $C M$ theta series for some arithmetic $P$ with $k(P) \geq 2$.

The field $\widetilde{M}_{V, r}(\mathcal{F})$ or $\widetilde{M}_{H, k}(\mathcal{F})$ is defined to be generated by the squares $\alpha_{l, P}^{2}$ and $\beta_{l, P}^{2}$ for the following reason. Suppose $\mathcal{F}$ has CM. Then, $f_{P}$ is a theta series of an imaginary quadratic field $M$ with even weight $k=k(P)$; so, $\rho_{P}=\operatorname{Ind}_{M}^{\mathbb{Q}} \lambda_{P}$ for a character $\lambda_{P}: \operatorname{Gal}(\overline{\mathbb{Q}} / M) \rightarrow \overline{\mathbb{Q}}_{p}^{\times}$. If $l$ is inert in $M$, writing $\mathfrak{l}$ for the unique prime of $M$ over $l$, we have

$$
\begin{aligned}
\operatorname{det}\left(X-\rho_{P}\left(F_{r o b}\right)\right)=\operatorname{det}\left(X-\operatorname{Ind}_{M}^{\mathbb{Q}} \lambda_{P}\left(F r o b_{l}\right)\right) & \\
& =X^{2}-\lambda_{P}\left(F r o b_{l}\right)=X^{2}+\psi_{k} \varepsilon_{P}(l) l^{k-1} .
\end{aligned}
$$

Thus $\alpha_{l}= \pm \sqrt{-\psi_{k} \varepsilon_{P}(l) l^{k-1}}$. Since $k-1$ is odd, $\mathbb{Q}\left(\alpha_{l} \mid l\right.$ :inert $)$ contains infinitely many distinct quadratic extensions $\mathbb{Q}(\sqrt{-l})$ for primes $l$ with $\psi_{k} \varepsilon_{P}(l)=1$. To avoid this, we need to take the square $\alpha_{l}^{2}$ in the vertical case and in the horizontal case if $k(P)$ is even.

\section{Hecke Fields AND their QUAdRatic EXTEnsions}

The "only if" part of Proposition 3.5 directly follows from the following lemma, though we will give a detailed proof of the proposition after proving the lemma.

Lemma 4.1. Let $f \in S_{k}\left(\Gamma_{0}(N), \psi\right)$ be a Hecke eigenform for $k \geq 2$. Let $\mathcal{P}$ be the set of all prime ideals of $\mathbb{Q}(f)$ split over $\mathbb{Q}$ (which has density 1 in the total set of prime ideals of $\mathbb{Q}(f)$ ). If $f$ does not have $C M$ (that is, it is not a theta series of an imaginary quadratic field), the field generated by the squares of Frobenius eigenvalues $\alpha_{l}$ and $\beta_{l}$ for $l$ running through any density 1 subset $\mathcal{P}_{0}$ of $\mathcal{P}$ is an infinite extension of $\mathbb{Q}(f)$. Here the density is computed in the set of all primes of $\mathbb{Q}(f)$.

Proof. For a prime $\mathfrak{l}$ of $\mathbb{Q}(f)$, write $\rho_{\mathfrak{l}}$ for the $\mathfrak{l}$-adic Galois representation of $f$. Write $O \subset \mathbb{Q}(f)$ for the integer ring of $\mathbb{Q}(f)$. Then we consider the adelic representation $\widehat{\rho}=\prod_{\mathfrak{l}} \rho_{\mathfrak{l}}: \operatorname{Gal}(\overline{\mathbb{Q}} / \mathbb{Q}) \rightarrow G L_{2}(\widehat{O})$ for $\widehat{O}=\prod_{\mathfrak{l}} O_{\mathfrak{l}}$. By a result of Ribet (see $\left.\underline{\mathbb{R}}\right)$, $\operatorname{Im}(\widehat{\rho})$ contains an open subgroup $U$ of $S L_{2}(\widehat{\mathbb{Z}})$ for $\widehat{\mathbb{Z}}=\prod_{l} \mathbb{Z}_{l}$. Let $S_{1}$ be the finite set of primes $l$ such that $U \cap S L_{2}\left(\mathbb{Z}_{l}\right) \neq S L_{2}\left(\mathbb{Z}_{l}\right)$ and let $\mathcal{S} \subset \mathcal{P}_{0}$ be a finite set outside $S_{1}$. Since $\mathfrak{l} \in \mathcal{P}_{0}$ for $\mathfrak{l} \in \mathcal{S}$, we have $O_{\mathfrak{l}}=\mathbb{Z}_{l}$ (for the residual characteristic $l=l(\mathfrak{l})$ of $\mathfrak{l}$ ). Let $S$ be the union of the set of residual characteristics of primes in $\mathcal{S}$, prime factors of $N p$ and $S_{1}$. Write $\rho_{\mathcal{S}}=\prod_{\mathfrak{l} \in \mathcal{S}} \rho_{\mathfrak{l}}$. Let $O_{\mathcal{S}}=\prod_{\mathfrak{l} \in \mathcal{S}} O_{\mathfrak{l}}$ and put $\mathbb{Q}_{\mathcal{S}}(f)=O_{\mathcal{S}} \otimes_{\mathbb{Z}} \mathbb{Q}$. Note that $\mathbb{Q}_{\mathcal{S}}(f)=\prod_{\mathfrak{r} \in \mathcal{S}} \mathbb{Q}_{l(\mathfrak{l})}$. We consider $H=\operatorname{Gal}(\overline{\mathbb{Q}} / \mathbb{Q}(f))$ which is an open subgroup of finite index in $\operatorname{Gal}(\overline{\mathbb{Q}} / \mathbb{Q})$. Since $\rho_{\mathcal{S}}$ is unramified outside $S \cup\{\infty\}$, we have well defined $\rho_{\mathcal{S}}\left(F r o b_{\mathfrak{l}}\right)$ for the Frobenius element $F r o b_{\mathfrak{l}}$ 
in $H$ for a prime ideal $\mathfrak{l}$ of $O$ outside $S$. Note that $F r o b_{\mathfrak{l}}=F r o b_{l(\mathfrak{l})}$ if $O_{\mathfrak{l}}=\mathbb{Z}_{l(\mathfrak{l})}$. Consider the commutative subalgebra $\mathbb{Q}\left[\rho_{\mathcal{S}}\left(F r o b_{\mathfrak{l}}\right)\right] \subset M_{2}\left(\mathbb{Q}_{\mathcal{S}}(f)\right)$ generated over $\mathbb{Q}$ by $\rho_{\mathcal{S}}\left(F r o b_{\mathfrak{l}}\right)$ for a prime ideal $\mathfrak{l}$ outside $S$ split in $\mathbb{Q}(f) / \mathbb{Q}$. Write $\mathbb{Z}\left[\rho_{\mathcal{S}}\left(F r o b_{\mathfrak{l}}\right)\right]$ for the subalgebra of $\mathbb{Q}\left[\rho_{\mathcal{S}}\left(F r o b_{\mathfrak{l}}\right)\right]$ generated over $\mathbb{Z}$ by $\rho_{\mathcal{S}}\left(F r o b_{\mathfrak{l}}\right)$. Consider the set $\mathcal{A}$ of tuples of (isomorphism classes of) commutative semi-simple quadratic extensions $A=\left\{A_{\mathfrak{l}} / \mathbb{F}_{l(\mathfrak{l})}\right\}_{\mathfrak{l} \in \mathcal{S}}$ indexed by primes in $\mathcal{S}$; so, $A_{\mathfrak{l}}$ is either a quadratic field extension of $\mathbb{F}_{l(\mathfrak{l})}$ or $\mathbb{F}_{l(\mathfrak{l})} \oplus \mathbb{F}_{l(\mathfrak{l})}$. Pick $t_{\mathfrak{l}} \in S L_{2}\left(\mathbb{Z}_{l(\mathfrak{l})}\right)$ for each $\mathfrak{l} \in \mathcal{S}$ such that

$$
\mathbb{Z}_{l(\mathfrak{l})}\left[t_{\mathfrak{l}}^{2}\right] \otimes_{\mathbb{Z}} \mathbb{F}_{l(\mathfrak{l})} \cong A_{\mathfrak{r}}
$$

Since $\mathcal{P}_{0}$ has density 1 among primes of $\mathbb{Q}(f)$, by Chebotarev density, we can find a split prime ideal $\mathfrak{l}=\mathfrak{l}(A) \in \mathcal{P}_{0}$ outside $S$ such that $\rho_{\mathcal{S}}\left(\right.$ Frob $\left._{\mathfrak{l}(A)}\right)$ is sufficiently close $\mathcal{S}$-adically to $t_{A}=\left(t_{\mathfrak{l}}\right)_{\mathfrak{r} \in \mathcal{S}} \in M_{2}\left(\mathbb{Q}_{\mathcal{S}}(f)\right)$ so that $\mathbb{Z}\left[\rho\left(F r o b_{\mathfrak{l}(A)}^{2}\right)\right] \otimes_{\mathbb{Z}} \mathbb{F}_{l(\mathfrak{l})} \cong A_{\mathfrak{l}}$ for each prime $\mathfrak{l} \in \mathcal{S}$. Note that $\mathbb{Q}_{A}:=\mathbb{Q}\left[\rho_{\mathcal{S}}\left(\operatorname{Frob}_{\mathfrak{l}(A)}^{2}\right)\right] \cong \mathbb{Q}\left(\alpha_{\mathfrak{l}(A)}^{2}\right)$, and for two distinct $A, A^{\prime} \in \mathcal{A}, \mathbb{Q}_{A} \neq \mathbb{Q}_{A^{\prime}}$. Thus at least we have found primes $\mathfrak{l}(A)$ indexed by $\mathcal{A}$ such that all quadratic extensions in $\left\{\mathbb{Q}\left(\alpha_{\mathfrak{r}(A)}^{2}\right)\right\}_{A \in A}$ of $\mathbb{Q}(f)$ are nonisomorphic. Making $|\mathcal{S}| \rightarrow \infty$, we can find an infinite set $\Omega \subset \mathcal{P}_{0}$ of primes $\mathfrak{l}$ such that $\mathbb{Q}\left(\alpha_{\mathfrak{r}}^{2}\right)$ are distinct for all $\mathfrak{l} \in \Omega$. This shows the result.

Proof of $(W V)$. Pick $f_{P}$ in $\mathcal{F}$ without complex multiplication for an arithmetic $P$. Then by the above lemma, $\widetilde{M}_{V, r}(\mathcal{F}) \supset \mathbb{Q}\left(\alpha_{\mathfrak{l}}^{2} \mid \mathfrak{l} \in \Omega\right)$ and $\mathbb{Q}\left(\alpha_{\mathfrak{l}}^{2} \mid \mathfrak{l} \in \Omega\right)$ is an infinite extension of $\mathbb{Q}$, where $\Omega$ is as in the above proof of Lemma 4.1 .

Suppose now that $\mathcal{F}$ has complex multiplication by an imaginary quadratic extension $M / \mathbb{Q}$ in $\overline{\mathbb{Q}}$ as defined in (CM1). Let $\mathfrak{p}$ be the prime of $M$ induced by $\left.i_{p}\right|_{M}: M \hookrightarrow \overline{\mathbb{Q}}_{p}$. Then $\rho_{\mathbb{I}} \cong \operatorname{Ind}_{M}^{\mathbb{Q}} \lambda$ as in $(\mathrm{CM} 1)$, where $\lambda: \operatorname{Gal}(\overline{\mathbb{Q}} / M) \rightarrow \widetilde{\mathbb{I}}^{\times}$is a character unramified outside $\mathfrak{p} N$ for the normalization $\operatorname{Spec}(\widetilde{\mathbb{I}})$ of $\operatorname{Spec}(\mathbb{I})$. Write $\mathfrak{O}$ for the integer ring of $M$. We can forget about finitely many primes $\mathfrak{l}$ in a finite set $S$, as $\mathbb{Q}\left(\alpha_{\mathfrak{l}} \mid \mathfrak{l} \in S\right)$ is a finite extension of $\mathbb{Q}$. Take $S$ to be the set of ramified primes of $\rho_{\mathbb{I}}($ so, $S$ includes ramified primes in $M / \mathbb{Q})$. Thus for any prime $\mathfrak{l} \notin S$, it is either split or inert in $M / \mathbb{Q}$. If $(l)=\mathfrak{l} \mathfrak{l}$ in $M$ for primes $\mathfrak{l} \neq \overline{\mathfrak{l}}, \alpha_{l}$ is the value of $\lambda_{P}\left(F r o b_{\mathfrak{l}}\right)=\lambda\left(F r o b_{\mathfrak{l}}\right) \bmod P$ for a prime $\mathfrak{l}$ in $M$ over $l$. If $(l)=\mathfrak{l}$ is inert in $M / \mathbb{Q}, \alpha_{l}^{2}=\lambda_{P}\left(F_{r o b}\right)$ as $\operatorname{det}\left(X-\rho_{P}\left(F_{r o b}\right)\right)=X^{2}-\lambda_{P}\left(F r o b_{\mathfrak{l}}\right)$. Let $\mathbb{F}$ be the residue field of $\widetilde{\mathbb{I}}$ (note that $\widetilde{\mathbb{I}}$ is a local ring with maximal ideal $\mathfrak{m}$, because it is finite flat over $\Lambda$ ). Write $W$ for the ring of Witt vectors of $\mathbb{F}$; so, $W$ is a finite flat discrete valuation ring unramified over $\mathbb{Z}_{p}$. Let $\left(R, \widetilde{\lambda}: \operatorname{Gal}(\overline{\mathbb{Q}} / M) \rightarrow R^{\times}\right)$be the universal couple with the universal character unramified outside $\mathfrak{p} N$ deforming $(\lambda \bmod \mathfrak{m})$ over $W$. This couple $(R, \tilde{\lambda})$ is characterized by the following universal property: For any local Artinian $W$-algebra $A$ with residue field $\mathbb{F}$ and any character $\varphi: \operatorname{Gal}(\overline{\mathbb{Q}} / M) \rightarrow A^{\times}$unramified outside $\mathfrak{p} N$ with $\varphi \bmod \mathfrak{m}_{A}=\lambda \bmod \mathfrak{m}$ for the maximal ideal $\mathfrak{m}_{A}$ of $A$, there exists a unique $W$-algebra homomorphism $\iota: R \rightarrow A$ such that $\varphi=\iota \circ \widetilde{\lambda}$. The pair $(A, \varphi)$ is called a deformation of $\lambda$ (see $[\mathrm{M}]$ ).

Writing $\widetilde{\Gamma}$ for the $p$-primary part of $C l_{M}\left(\mathfrak{p}^{\infty} N\right)=\varliminf_{n} C l_{M}\left(\mathfrak{p}^{n} N\right)$ (for the ray class groups $C l_{M}\left(\mathfrak{p}^{n} N\right)$ modulo $\mathfrak{p}^{n} N$ of $\left.M\right)$, we have $R \cong W[[\widetilde{\Gamma}]]$ by class field theory. To see this, we pick a deformation $\varphi: \operatorname{Gal}(\overline{\mathbb{Q}} / M) \rightarrow A^{\times}$of $\lambda$ unramified outside $\mathfrak{p} N$; thus, $A$ is a local Aritinian $W$-algebra sharing the residual field with 
$W$ and $\varphi \bmod \mathfrak{m}_{A}=\lambda \bmod \mathfrak{m}$ for the maximal ideal $\mathfrak{m}_{A}$ of $A$. Let $\lambda_{0}$ be the Teichmüller lift of $\lambda \bmod \mathfrak{m}$; so, $\varphi^{\prime}=\varphi \lambda_{0}^{-1}$ has $p$-power order. For a prime $l \mid N$, taking a prime factor $\mathfrak{l}$ of $l$ in $M$, by class field theory, the image $I_{\mathfrak{l}}^{a b}$ of the inertia group $I_{\mathfrak{r}} \subset \operatorname{Gal}(\overline{\mathbb{Q}} / M)$ in the Galois group of the maximal abelian extension of $M$ over $M$ is isomorphic to the multiplicative group $\mathfrak{O}_{\mathfrak{l}}^{\times}$of the $\mathfrak{l}$-adic integer ring of $M_{\mathfrak{l}}$. Since $\varphi^{\prime}$ has $p$-power order and $p \neq l, \varphi^{\prime}$ must be trivial on $1+\mathfrak{O}_{\mathfrak{l}} \subset \mathfrak{O}_{\mathfrak{l}}^{\times}$. Thus the $\mathfrak{l}$-conductor of $\varphi^{\prime}$ is at most $\mathfrak{l}$, and hence $\varphi=\varphi^{\prime} \lambda_{0}$ factors through $C l_{M}\left(\mathfrak{p}^{\infty} N\right)$. Thus $\varphi^{\prime}$ factors through the maximal $p$-profinite quotient $\widetilde{\Gamma}$ and extends to a unique $W$-algebra homomorphism $\iota=\iota_{\varphi}: W[[\widetilde{\Gamma}]] \rightarrow A$ such that $\left.\iota\right|_{\widetilde{\Gamma}}=\varphi^{\prime}$. Since $\widetilde{\Gamma}$ is the maximal $p$-profinite quotient of $C l_{M}\left(\mathfrak{p}^{\infty} N\right)$, by class field theory, we have the corresponding subfield $\widetilde{M}$ of the ray class field modulo $\mathfrak{p}^{\infty} N$ with $\operatorname{Gal}(\widetilde{M} / M) \cong \widetilde{\Gamma}$ by the Artin symbol. Writing the inclusion $\widetilde{\Gamma} \subset W[[\widetilde{\Gamma}]]$ as $\gamma \mapsto[\gamma]$ and identifying $\operatorname{Gal}(\widetilde{M} / M)=\widetilde{\Gamma}$, define a character $\widetilde{\lambda}: \operatorname{Gal}(\overline{\mathbb{Q}} / M) \rightarrow W[\widetilde{\Gamma}]]$ by $\widetilde{\lambda}(\sigma)=\lambda_{0}(\sigma)\left[\left.\sigma\right|_{\widetilde{M}}\right]$. Then by our construction $\iota \circ \widetilde{\lambda}=\varphi$; so, $(W[\widetilde{\Gamma}]], \widetilde{\lambda})$ satisfies the universal property of $(R, \tilde{\lambda})$ for deformations $\varphi$ of $\lambda$.

Let $\Delta \subset \widetilde{\Gamma}$ be the maximal torsion subgroup of $\widetilde{\Gamma}$. Then $\Delta$ is finite, and we put $\Gamma_{t f}=\widetilde{\Gamma} / \Delta$. We fix a splitting $\widetilde{\Gamma} \cong \Delta \times \Gamma_{t f} ;$ so, $R \cong W\left[\left[\Gamma_{t f}\right]\right][\Delta]$. Since $\Gamma_{t f}$ is a $p$ profinite cyclic group isomorphic to the additive group $\mathbb{Z}_{p}, W\left[\left[\Gamma_{t f}\right]\right]$ is isomorphic to a one-variable power series ring $W[[T]]$. Thus $\operatorname{Spec}(R)$ is equidimensional reduced, and its irreducible components are isomorphic to $\operatorname{Spec}(W[[T]])$.

By universality, we have a $W$-algebra homomorphism $\pi: R \rightarrow \widetilde{\mathbb{I}}$ such that $\pi \circ \widetilde{\lambda}=\lambda$. The image $\mathbb{I}^{\prime}$ of $\pi$ is the $\mathbb{I}$-subalgebra of $\widetilde{\mathbb{I}}$ topologically generated by $\lambda(\sigma)$ for all $\sigma \in \operatorname{Gal}(\overline{\mathbb{Q}} / M)$. Since $\operatorname{Spec}\left(\mathbb{I}^{\prime}\right)$ and $\operatorname{Spec}(R)$ has equal dimension, $\operatorname{Spec}\left(\mathbb{I}^{\prime}\right)$ is isomorphic to one of the irreducible components of $\operatorname{Spec}(R)$; so, $\pi$ induces $\operatorname{Spec}\left(\mathbb{I}^{\prime}\right) \cong \operatorname{Spec}(W[[T]])=\operatorname{Spec}\left(W\left[\left[\Gamma_{t f}\right]\right]\right)$. Since $\operatorname{Spec}(\widetilde{\mathbb{I}})$ is normalization of $\operatorname{Spec}(\mathbb{I})$ and $\operatorname{Spec}\left(\mathbb{I}^{\prime}\right) \cong \operatorname{Spec}\left(W\left[\left[\Gamma_{t f}\right]\right]\right.$ is regular, we have $\widetilde{\mathbb{I}}=\mathbb{I}^{\prime} \cong W\left[\left[\Gamma_{t f}\right]\right]$.

The inclusion $\mathbb{Z}_{p}^{\times} \hookrightarrow \mathfrak{O}_{\mathfrak{p}}^{\times}$induces an inclusion $\Gamma \rightarrow C l_{M}\left(\mathfrak{p}^{\infty} N\right)$. Composing this inclusion with the projection: $C l_{M}\left(\mathfrak{p}^{\infty} N\right) \rightarrow \Gamma_{t f}$, we regard $\Gamma$ as a subgroup of $\widetilde{\Gamma}_{t f}$ of finite index. This $W[[\Gamma]]$-algebra structure of $\mathbb{I}=W\left[\left[\Gamma_{t f}\right]\right]$ is equal to that coming from the inclusion $W[[\Gamma]] \hookrightarrow \mathbb{I}$ given by $\gamma \mapsto(1+X)$ for the variable $X$ not $x$. This is because a Hecke character of infinity type $k-1$ gives rise to its theta series of weight $k$. Then for an arithmetic point $P$ with $r(P) \leq r, \lambda_{P}=P \circ \lambda$ has infinity type $k(P)-1$; that is, $\lambda_{P}(\alpha)=\alpha^{k(P)-1}$ for $\alpha \in M$ congruent to 1 modulo $N p^{r}$. Thus for the class number $h$ of $M$, taking a generator $\alpha$ of $\mathfrak{l}^{h}$, we have $\lambda_{P}(\mathfrak{l})=\alpha^{(1 / h)(k(P)-1)} \zeta$ for $\zeta \in \mu_{p^{r} h}$. In other words, choosing a complete representative set $\left\{\mathfrak{a}_{j}\right\}_{j=1, \ldots, h}$ of ideal classes of $M$, taking a generator $\alpha_{j}$ of $\mathfrak{a}_{j}^{h}$ and writing $K_{r}=\mathbb{Q}\left(\mu_{p^{r} h}\right)\left[\alpha_{j}^{1 / h} \mid j=1, \ldots, h\right]$, we find that $\mathbb{Q}\left(\alpha_{l}^{2}\right) \subset K_{r}$, which is a finite extension of $\mathbb{Q}$ independent of $P$ (as long as $r(P) \leq r$ ); so, $\widetilde{M}_{V, r} \subset K_{r}$. This finishes the proof.

Proof of (WH). By the above proof of (WV), if $\mathcal{F}$ has complex multiplication, $\widetilde{M}_{H, r}(\mathcal{F}) \subset K_{\infty}=\bigcup_{r} K_{r}=\mathbb{Q}\left(\mu_{p^{\infty}}, \mu_{h}\right)\left[\alpha_{j}^{1 / h} \mid j=1, \ldots, h\right]$, which is a finite extension of $\mathbb{Q}\left(\mu_{p^{\infty}}\right)$.

In the proof of Lemma 4.1, for a non-CM form $f$, we constructed an infinite set $\Omega$ of primes such that $\left\{\mathbb{Q}(f)\left(\alpha_{l(\mathfrak{l})}^{2}\right)\right\}_{\mathfrak{l} \in \Omega}$ is an infinite set of distinct quadratic extensions of $\mathbb{Q}(f)$. Since $L:=\mathbb{Q}(f)\left[\mu_{p^{\infty}}\right]$ has only finitely many distinct quadratic 
extensions of $\mathbb{Q}(f)$ inside, the extension $L\left(\alpha_{l(\mathfrak{l})}^{2} \mid \mathfrak{l} \in \Omega\right) / L$ still has infinite dimension over $\mathbb{Q}\left[\mu_{p} \infty\right]$. Thus the result follows, taking $f=f_{P}$ for an arithmetic member $f_{P}$ $(k(P)=k)$ of a non-CM family $\mathcal{F}$.

We have the following corollary to the above proofs of (WV) and (WH):

Corollary 4.2. Let the notation be as in the previous section. If $\mathcal{F}$ is a CM family, then

$$
\left[M_{H, \mathcal{A}}(\mathcal{F}): \mathbb{Q}\left(\mu_{p^{\infty}}\right)\right]<\infty \text { and }\left[M_{V, \mathcal{A}}(\mathcal{F}): \mathbb{Q}\right]<\infty
$$

where $\mathcal{A}$ is an infinite set of arithmetic points for a fixed weight $k \geq 2$ in the horizontal case and of bounded p-power level $p^{r} \mathbf{p}$ in the vertical case.

Proof. The necessity of taking the square $\alpha_{l}^{2}$ in the above proof of (WV) and (WH) comes from the inertness of the prime $l$ in $M$ (and also the fact that we vary the primes $l$ in an infinite set). Since $p$ is split in $M$, we have $\alpha_{p, P}=\lambda_{P}\left(F r o b_{\mathfrak{p}}\right)$ for a factor $\mathfrak{p}$ of $p$ in $M$, and under the notation of the proof of (WV), we find that $M_{V, \mathcal{A}}(\mathcal{F}) \subset K_{r}$, since the prime $p$ splits in $M$. The horizontal case can be proven similarly, because $M_{H, \mathcal{A}}(\mathcal{F}) \subset K_{\infty}$.

We add one more lemma:

Lemma 4.3. Let $\mathcal{F}$ be a slope 0 p-adic analytic family of Hecke eigenforms with coefficients in $\mathbb{I}$. Then we have:

(1) Fix $0 \leq r<\infty$. Then the degree $\left[\mathbb{Q}\left(f_{P}\right): \mathbb{Q}\left(a\left(p, f_{P}\right)\right)\right]$ for arithmetic $P$ with $r(P) \leq r$ is bounded independently of $P$.

(2) Let $K=\mathbb{Q}\left(\mu_{p^{\infty}}\right)$ and fix $k \geq 2$. Then the degree $\left[K\left(f_{P}\right): K\left(a\left(p, f_{P}\right)\right)\right]$ for arithmetic $P$ with $k(P)=k$ is bounded independently of $P$.

Proof. Since the proof is basically the same, we prove (1). We prove that the index $\left[L\left(f_{P}\right): L\left(a\left(p, f_{P}\right)\right)\right]$ is bounded independently of $P$ for $L=\mathbb{Q}\left(\mu_{p^{r}}\right)$. Let $\Gamma_{r}=\Gamma_{1}(N \mathbf{p}) \cap \Gamma_{0}\left(p^{r} \mathbf{p}\right)$. Then by the control theorem as in (C1-2) in Section 1 , $\operatorname{dim}_{\mathbb{C}_{p}} S_{k}^{\text {ord }}\left(\Gamma_{r}, \varepsilon ; \mathbb{C}_{p}\right)$ is a constant $d$ independent of $k$ and $r$, where $S_{k}\left(\Gamma_{r}, \varepsilon ; A\right)$ is the space of modular forms with coefficients in $A$ with character $\varepsilon: \Gamma / \Gamma^{p^{r}} \rightarrow A^{\times}$. Since $\varepsilon$ has values in $\mu_{p^{r}}\left(\mathbb{C}_{p}\right)$, if $\sigma \in \operatorname{Aut}\left(\mathbb{C}_{p} / L\left(a\left(p, f_{P}\right)\right)\right)$, then $f_{P}^{\sigma}$ is another Hecke eigenform within the same space $S_{k}^{\text {ord }}\left(\Gamma_{r}, \varepsilon_{P} ; \mathbb{C}_{p}\right)$. Thus

$$
\left[L\left(f_{P}\right): L\left(a\left(p, f_{P}\right)\right)\right]=\#\left\{f_{P}^{\sigma} \mid \sigma \in \operatorname{Aut}\left(\mathbb{C}_{p} / L\left(a\left(p, f_{P}\right)\right)\right)\right\} \leq d,
$$

as desired.

\section{Results towards the STRONG HORIZONTAL THEOREM}

We start with

Lemma 5.1. Let $W \subset \overline{\mathbb{Q}}_{p}$ be a valuation ring finite flat over $\mathbb{Z}_{p}$ with quotient field $F$. Let $\Phi(T) \in W[[T]]$, and suppose that there is an infinite subset $\Omega \subset \mu_{p}\left(\overline{\mathbb{Q}}_{p}\right)$ such that $\Phi(\zeta-1) \in \mu_{p^{\infty}}\left(\overline{\mathbb{Q}}_{p}\right)$ for all $\zeta \in \Omega$. Then there exists $\zeta_{0} \in \mu_{p^{\infty}}(W)$ and $s \in \mathbb{Z}_{p}$ such that $\zeta_{0}^{-1} \Phi(T)=(1+T)^{s}=\sum_{n=0}^{\infty}\left(\begin{array}{l}s \\ n\end{array}\right) T^{n}$.

We shall give two proofs of the lemma. The second more elementary one is due to Kiran Kedlaya.

First proof. We use the following lemma of Chai (see [Ch1, Theorem 4.2], Ch2, Theorem 6.6] and [ $\mathrm{H10}$, Theorem 3.6]), which in the simplest case can be stated as 
Rigidity Lemma. Let $Z$ be an integral connected formal subscheme $Z$ of codimension 1 containing the identity of $\left.\widehat{\mathbb{G}}_{m} \times \widehat{\mathbb{G}}_{m / \overline{\mathbb{F}}_{p}}=\operatorname{Spf}\left(\overline{\mathbb{F}}_{p} \widehat{\left[t, t^{-1}\right]}\right) \times \operatorname{Spf}\left(\overline{\mathbb{F}_{p}} \widehat{\left[t^{\prime}, t^{\prime}\right.}-1\right]\right)$. If $Z$ is stable under the diagonal action $\left(t, t^{\prime}\right) \mapsto\left(t^{z}, t^{\prime z}\right)$ for all $z$ in an open subgroup $U$ of $\mathbb{Z}_{p}^{\times}$, then $Z$ is a formal subtorus of codimension 1 .

Making the variable change $T \mapsto \zeta_{1}^{-1}(T+1)-1$ for a $\zeta_{1} \in \Omega$ (replacing $W$ by its finite extension if necessary), we may replace $\Omega$ by $\zeta_{1}^{-1} \Omega \ni 1$; so, rewriting $\zeta_{1}^{-1} \Omega$ as $\Omega$, we may assume that $1 \in \Omega$. Then $\Phi(0)=\zeta_{0} \in \mu_{p^{\infty}}$. Thus again replacing $\Phi$ by $\zeta_{0}^{-1} \Phi$, we may assume that $\Phi(0)=1$.

For $\sigma \in \operatorname{Gal}\left(F\left(\mu_{p^{\infty}}\right) / F\right)$ with the quotient field $F$ of $W, \Phi\left(\zeta^{\sigma}-1\right)=\Phi(\zeta-1)^{\sigma}$. Writing $\phi(\zeta)=\Phi(\zeta-1)$, the above identity yields $\phi\left(\zeta^{\sigma}\right)=\phi(\zeta)^{\sigma}$. Identifying $\operatorname{Gal}\left(F\left(\mu_{p^{\infty}}\right) / F\right)$ with an open subgroup $\Gamma$ of $\mathbb{Z}_{p}^{\times}$and writing $\sigma_{z} \in \operatorname{Gal}\left(F\left(\mu_{p^{\infty}}\right) / F\right)$ for the element corresponding to $z \in \Gamma$, we find that

$$
\Phi \circ z(\zeta-1)=\Phi\left(\zeta^{z}-1\right)=\Phi\left(\zeta^{\sigma_{z}}-1\right)=\Phi(\zeta-1)^{\sigma_{z}}=z \circ \Phi(\zeta-1) .
$$

We find that $z \circ \phi=\phi \circ z$ is valid on the Zariski dense subset $\Omega$ of $\operatorname{Spec}(W[[T]])$; so, $\phi$ as a scheme endomorphism of $\widehat{\mathbb{G}}_{m}$ commutes with the action of $z$.

Regard $W[[T]]$ as the affine ring of the formal torus $\widehat{\mathbb{G}}_{m / W}=\operatorname{Spf}\left(\widehat{W\left[t, t^{-1}\right.}\right]$ ) (so that $T$ is given by $t-1)$. Note that $z \in \mathbb{Z}_{p}^{\times}$acts on $\widehat{\mathbb{G}}_{m}$ as a group automorphism induced by a $W$-bialgebra automorphism of $W[[T]]$ sending $t=(1+T) \mapsto t^{z}=$ $(1+T)^{z}$. Take $\phi \in \operatorname{End}_{W \text {-SCH }}\left(\widehat{\mathbb{G}}_{m}\right)$ sending 1 to 1 . Put $\widehat{\mathbf{G}}:=\widehat{\mathbb{G}}_{m} \times \widehat{\mathbb{G}}_{m / W}$. We consider the graph $\Gamma_{\phi}$ of $\phi$, which is an irreducible formal subscheme $\Gamma_{\phi} \subset \widehat{\mathbb{G}}_{m} \times \widehat{\mathbb{G}}_{m}$ smooth over $W$. Writing the variable on $\widehat{\mathbf{G}}$ as $\left(T, T^{\prime}\right), \Gamma_{\phi}$ is the closed formal subscheme defined by the principal ideal $\left(t^{\prime}-\phi(t)\right)$. If $\phi \circ z=z \circ \phi$ for all $z$ in an open subgroup $U$ of $\operatorname{Aut}_{g p}\left(\widehat{\mathbb{G}}_{m}\right)=\mathbb{Z}_{p}^{\times}$, then $\Gamma_{\phi}$ is stable under the diagonal action of $U$ on $\widehat{\mathbf{G}}$. Then, extending the scalar from $W$ to the composite $\widetilde{W}$ of $W$ and the ring $W\left(\overline{\mathbb{F}}_{p}\right)$ of Witt vectors with coefficients in the algebraic closure $\overline{\mathbb{F}}_{p}$ of $\mathbb{F}_{p}$, by the rigidity lemma, we find that the reduction modulo $\mathfrak{m}_{\widetilde{W}}$ of $\Gamma_{\phi}$ is a formal subtorus of $\widehat{\mathbf{G}}_{/ \overline{\mathbb{F}}_{p}}$.

We regard $\Phi$ as an endomorphism $\phi$ of $W[[T]]$ sending $1+T$ to $\Phi(T)$. In other words, $f(T)^{\phi}=f(\Phi(T)-1)$. Applying the above argument to the morphism $\phi$, we find $\Phi(T) \equiv(1+T)^{s} \bmod \mathfrak{m}_{\widetilde{W}}$ for some $s \in \mathbb{Z}_{p}$. The quotient $\Phi_{1}(T)=$ $\Phi(T) /(1+T)^{s} \in W[[T]]$ again satisfies $\Phi_{1}\left(t^{z}\right)=\Phi_{1}(t)^{z}$ for $z \in U$ and $\Phi_{1}(0)=1$. Then by the same argument as above, $\Phi_{1}(T) \equiv(1+T)^{s_{1}} \bmod \mathfrak{m}_{\widetilde{W}}$ for some $s_{1} \in \mathbb{Z}_{p}$. On the other hand, $(1+T)^{s_{1}} \equiv \Phi_{1}(T)=1 \bmod \mathfrak{m}_{\widetilde{W}}$ in $\overline{\mathbb{F}}_{p}[[T]]$, which shows that $s_{1}=0$. Since $\Phi(\zeta-1) \in \mu_{p^{\infty}}$ for $\zeta \in \Omega, \Phi_{1}(\zeta-1) \equiv 1 \bmod \mathfrak{m}_{W}$. Since there are only finitely many $p$-power roots of unity congruent to 1 modulo $\mathfrak{m}_{\widetilde{W}}, \Phi_{1}$ is constant. Since $\Phi_{1}(0)=1$, we get $\Phi_{1}=1$, and $\Phi(T)=(1+T)^{s}$, as desired. This fact is mentioned in [Ch2, Remark 6.6.1 (iv) after Theorem 6.6].

Second proof (Kiran Kedlaya). As we have remarked above, we may assume that $1 \in \Omega$ and $\Phi(0)=1$. Note that $t=1 \Leftrightarrow T=0$. Thus we are trying to show that $\Phi(T)=(1+T)^{s}$ for some $s \in \mathbb{Z}_{p}$. In this proof, the residue field $\mathbb{F}$ of $W$ is a finite extension of $\mathbb{F}_{p}$.

Write the valuation of $W$ as $v$ (and use the same symbol $v$ for an extension of $v$ to $W\left[\mu_{p^{\infty}}\right]$ ). Normalize $v$ so that $v(p)=1$. Suppose that $\Phi(T) \notin W$ (nonconstant). Write $\Phi(T)-1=\sum_{i=1}^{\infty} a_{i} T^{i}$. Since $W$ is a discrete valuation ring, there is a least 
index $j>0$ for which $v\left(a_{j}\right)$ is minimized. For $\epsilon$ sufficiently small, if $v(\tau)=\epsilon$, then $v(\Phi(\tau)-1)=v\left(a_{j}\right)+j \epsilon$. In particular, for $\zeta$ a $p$-power root of unity, taking $\tau=\zeta-1$, we have $v(\zeta-1)=p^{-m} /(p-1)$ for some nonnegative integer $m$, so we have infinitely many relations of the form $j p^{-m} /(p-1)+v\left(a_{j}\right)=p^{-n} /(p-1)$. Then, we have $m \rightarrow \infty \Rightarrow n \rightarrow \infty$ (by continuity and nonconstancy of $\tau \mapsto \Phi(\tau)$ ); so, taking limits under $m \rightarrow \infty$ yields $v\left(a_{j}\right)=0$. Also, $j$ must be a power of $p$, say $j=p^{h}$, and for $m$ large we have $n=m-h$.

Since $v\left(a_{j}\right)=0, a_{j} \bmod \mathfrak{m}_{W}$ is in $\mathbb{F}^{\times}$. For the moment, assume $\mathbb{F}=\mathbb{F}_{p}$. That is, $a_{j}$ reduces to an integer $b_{0}$ coprime to $p$ in the residue field of $W$. We can thus replace $\Phi(T)$ by $\Phi_{1}(T)$ defined by $\Phi(T)=\Phi_{1}(T) \times(1+T)^{s}$ for some $s$ (namely $s=b_{0} j=b_{0} p^{h_{0}}$ for $\left.h_{0}:=h\right)$ so as to increase the least index $j$ for which $v\left(a_{j}\right)=0$. Indeed, writing $\Phi(T)=\sum_{n=0}^{j} a_{n} T^{n}+T^{j+1} f(T)$ with $f(T) \in W[[T]]$, we have

$$
\sum_{n=0}^{j} a_{n} T^{n} \equiv 1+b_{0} T^{p^{h_{0}}} \equiv\left(1+T^{p^{h_{0}}}\right)^{b_{0}} \equiv(1+T)^{s} \quad \bmod \left(\mathfrak{m}_{W}+\left(T^{j+1}\right)\right) .
$$

We have $\Phi_{1}(T) \equiv 1+T^{j+1} f(T)(1+T)^{-s} \equiv 1 \bmod \left(\mathfrak{m}_{W}+\left(T^{j+1}\right)\right)$. Thus if we write $j_{1}$ for the $j$ for this new $\Phi_{1}$, then $j_{1}>j$, and $j_{1}=p^{h_{1}}$ with $h_{1}>h_{0}$ and $a_{j_{1}} \equiv b_{1} \bmod \mathfrak{m}_{W}$ for $b_{1} \in \mathbb{Z}$. Repeating this, for $s=\sum_{k=0}^{\infty} b_{k} p^{h_{k}} \in \mathbb{Z}_{p}$,

$$
\frac{\Phi(T)}{(1+T)^{s}}-1=\sum_{n=1} a_{n} T^{n}
$$

no longer has a least $j$ with minimal $v\left(a_{j}\right)$; so, $\Phi(T) /(1+T)^{s}=1$, and we get $\Phi(T)=(1+T)^{s}$.

Suppose now that $\mathbb{F} \neq \mathbb{F}_{p}$. We have the Frobenius automorphism $\phi$ fixing $\mathbb{Z}_{p}\left[\mu_{p^{\infty}}\right] \subset W\left[\mu_{p^{\infty}}\right]$. Letting $\phi$ acts on power series by $\left(\sum_{n} a_{n} T^{n}\right)^{\phi}=\sum_{n} a_{n}^{\phi} T^{n}$, we find $\Phi^{\phi}\left(t^{\phi}\right)=\Phi(t)^{\phi}$. Since $\Phi(\zeta-1)$ is a $p$-power root of unity for $\zeta$ in an infinite set $\Omega \subset \mu_{p^{\infty}}$, we have $\Phi^{\phi}(\zeta-1)=\Phi^{\phi}\left(\zeta^{\phi}-1\right)=\Phi(\zeta-1)^{\phi}=\Phi(\zeta-1)$. Since $\Omega \subset \widehat{\mathbb{G}}_{m}$ is Zariski dense, we find that $\Phi^{\phi}=\Phi$, which shows that $\Phi \in W^{\phi}[[T]]$ for the subring $W^{\phi}$ fixed by $\phi$. Note that the residue field of $W^{\phi}$ is $\mathbb{F}_{p}$, and the earlier argument applies to $\Phi \in W^{\phi}[[T]]$.

Take a prime $l$ with $\alpha_{l, P} \neq 0$ for some $P$. If $l \mid N p$, put $A=a(l)$ (the image of $U(l)$ in $\mathbb{I})$, and otherwise, fix a root $A$ of $\operatorname{det}\left(T-\rho_{\mathbb{I}}\left(F r o b_{l}\right)\right)=0$. Replacing $\mathbb{I}$ by its finite extension, we assume that $A \in \mathbb{I}$. Recall that $A_{P}=P(A)$. Let $X=\gamma^{-1}(1+x)-1$ be the variable of $\Lambda=W[[\Gamma]]$ centered at weight 1 . Let $Q$ be the quotient field of $\Lambda$ and fix its algebraic closure $\bar{Q}$. We embed $\mathbb{I}$ into $\bar{Q}$ and regard it as a subring of $\bar{Q}$. Take the $p^{n}$-th root $X^{1 / p^{n}}$ of $X$ in $\bar{Q}$ and consider $W\left[\mu_{p^{n}}\right][[X]]\left[X^{1 / p^{n}}\right] \subset \bar{Q}$ which is independent of the choice of $X^{1 / p^{n}}$.

Recall, as explained after Conjecture 3.4 that if a prime $l$ is a factor of $N$ (so $l \neq p$ ) and if $f_{P}$ (or more precisely the automorphic representation generated by $f_{P}$ ) is Steinberg (resp. super-cuspidal) at $l$ for an arithmetic point $P$, then all members of $\mathcal{F}$ are Steinberg (resp. super-cuspidal) at $l$. Since $\alpha_{l, P} \neq 0$ for some $P, f_{P}$ is not super-cuspidal at $l$ for any arithmetic $P$.

Proposition 5.2. Let the notation be as above and write $K:=\mathbb{Q}\left[\mu_{p^{\infty}}\right]$ and $L_{P}=$ $K\left(A_{P}\right)$ for each arithmetic point $P$ with $k(P)=k$. Fix a rational prime $l$. Suppose that there exists an infinite set $\mathcal{A}$ of arithmetic points with $k(P)=k \geq 2$ satisfying 
one of the following two conditions:

(i) $L_{P} / K$ is a finite extension of bounded degree independently of $P \in \mathcal{A}$, and in $L_{P} / K$, the prime $l$ is at worst tamely ramified for all $P \in \mathcal{A}$.

(ii) The composite of $L_{P}$ for all $P \in \mathcal{A}$ is a finite extension of $\mathbb{Q}\left[\mu_{p} \infty\right]$.

Then we have $A \in W\left[\mu_{p^{n}}\right][[X]]\left[(1+X)^{1 / p^{n}}\right] \cap \mathbb{I}$ in $\bar{Q}$ for $0 \leq n \in \mathbb{Z}$, and we have the following two possibilities:

(1) There exist a Weil l-number $\alpha_{1}$ of weight 1 and a root of unity $\zeta_{0}$ such that $A_{P}=\alpha_{l, P}=\zeta_{0}\left\langle\alpha_{1}\right\rangle^{k(P)-1}$; in other words, $A(X)=\zeta_{0}(1+X)^{s}$ for $s=\frac{\log _{p}\left(\alpha_{1}\right)}{\log _{p}(\gamma)}$ with the Iwasawa $p$-adic logarithm $\log _{p}$. This is the case where the automorphic representation generated by $f_{P}$ for an arithmetic point $P$ with $k(P)>2$ or with $\varepsilon_{P} \neq 1$ is in the principal series at $l$.

(2) We have $l \neq p$, and there exists a root of unity $\zeta_{0}$ such that $A(X)=$ $\zeta_{0} l^{-1 / 2}(1+X)^{s}$ for $s=\frac{\log _{p}(l)}{2 \log _{p}(\gamma)}$. This is the case where the automorphic representation generated by $f_{P}$ for an arithmetic points $P$ is Steinberg at $l \neq p$.

Proof. We give a proof assuming (i), since the proof of the other case is almost identical using Lemma 2.3 in place of Corollary 2.5. By Corollary 2.5, we have only a finite number of Weil $l$-numbers of weight $k$ in $\bigcup_{P \in \mathcal{A}} L_{P}$ up to multiplication by roots of unity, and hence $A_{P}$ for $P \in \mathcal{A}$ hits one of such Weil $l$-numbers $\alpha$ of weight $k-1$ infinitely many times, up to roots of unity, unless the automorphic representation generated by $f_{P}$ is Steinberg at $l \neq p$. If $f_{P_{0}}$ is Steinberg at $l \neq p$ for one arithmetic $P_{0}, f_{P}$ is Steinberg for all arithmetic $P$. This fact, as already explained, follows either from the $\Lambda$-adic version of the theory of new forms (cf. [H88, Theorem 3.6]) or from the rigidity of local Galois deformations at $l$ in characteristic 0 (cf. [HMI, Theorem 3.75]) or from the fact that we can shift the family $\mathcal{F}$ to automorphic forms on a quaternion algebra ramified at $l$ by the Jacquet-Langlands correspondence (cf. [H00, Section 7]). Thus $A_{P}=l^{-1 / 2} l^{(k(P)-1) / 2}$ up to roots of unity for all $P \in \mathcal{A}$ (see [MFM], Theorem 4.6.17]). In this case, we put $\alpha=l^{(k-2) / 2}$.

Suppose for the moment that $\mathbb{I}=W[[X]]$ for a discrete valuation ring $W$ finite flat over $\mathbb{Z}_{p}$ (for the variable $X$ centered at weight 1 ); so, $n=0$. After a variable change $X \mapsto Y=\gamma^{1-k}(1+X)-1$, we have $\left.A(Y)\right|_{Y=0}=\left.A(X)\right|_{X=\gamma^{k-1}-1}$. Note that $|\alpha|_{p}=1$. Let $\Omega_{1}=\left\{\varepsilon_{P}(\gamma) \mid P \in \mathcal{A}\right\}$, which is an infinite set in $\mu_{p}(K)$. Let $\Phi_{1}(Y):=\alpha^{-1} A(Y)=A\left(\gamma^{1-k}(1+X)-1\right) \in W[[Y]]$ and $\mathbf{L}$ be the composite of $L_{P}$ for $P$ running through $\mathcal{A}$. The subset $\Omega_{2}$ of $\Omega_{1}$ made up of $\zeta \in \Omega_{1}$ such that $\Phi_{1}(\zeta-1)$ is a root of unity in $\mathbf{L}$ is an infinite set. By Lemma 2.6, the group of roots of unity of $\mathbf{L}$ contains $\mu_{p^{\infty}}(K)$ as a subgroup of finite index, and we find an infinite subset $\Omega \subset \Omega_{2}$ and a root of unity $\zeta_{1}$ such that $\left\{\Phi_{1}(\zeta-1) \mid \zeta \in \Omega\right\} \subset \zeta_{1} \mu_{p^{\infty}}(K)$. Then $\Phi=\zeta_{1}^{-1} \Phi_{1}$ satisfies the assumption of Lemma 5.1 and for a root of unity $\zeta$, we have $A(Y)=\zeta \alpha(1+Y)^{s_{1}}$ for $s_{1} \in \mathbb{Z}_{p}$, and $A(X)=\zeta \alpha\left(\gamma^{1-k}(1+X)\right)^{s_{1}}$. Let $X=\zeta^{\prime} \gamma^{k^{\prime}-1}-1$ for $\zeta^{\prime} \in \mu_{p^{\infty}}(K)$. Then $A\left(\zeta^{\prime} \gamma^{k^{\prime}-1}-1\right)=\zeta \alpha\left(\zeta^{\prime} \gamma^{-k+k^{\prime}}\right)^{s_{1}}$, which is equal either to a Weil $l$-number of weight $k^{\prime}-1$ (the case of principal series at $l$ ) or to a root of unity $\zeta^{\prime \prime}$ times $l^{\left(k^{\prime}-2\right) / 2}$ (the case of Steinberg representation at $l$ ). In the latter case, taking the logarithm of $\zeta \alpha\left(\gamma^{-k+k^{\prime}}\right)^{s_{1}}=\zeta^{\prime \prime} l^{\left(k^{\prime}-2\right) / 2}$, we find

$$
s_{1}=\frac{\log _{p}(l)}{2 \log _{p}(\gamma)} .
$$


This shows that $l=p$ is impossible, since $f_{P}$ generates an automorphic representation principal at $p$ if $k(P)>2$ by $p$-ordinarity. In the former case, take $k^{\prime}>2$. Then

$$
\alpha_{1}:=\frac{A\left(\gamma^{k^{\prime}}-1\right)}{A\left(\gamma^{k^{\prime}-1}-1\right)}=\frac{\zeta \alpha\left(\zeta^{\prime} \gamma^{-k+k^{\prime}+1}\right)^{s_{1}}}{\zeta \alpha\left(\zeta^{\prime} \gamma^{-k+k^{\prime}}\right)^{s_{1}}}=\gamma^{s_{1}}
$$

which is an algebraic number $\alpha_{1}$ independent of $k^{\prime}$. Note that for $k^{\prime}>2, \alpha_{1}$ is a ratio of Weil $l$-numbers of weight $k^{\prime}-1$ and $k^{\prime}$, and hence $\alpha_{1}$ is not a root of unity. Thus we have $s_{1}=\frac{\log \left(\alpha_{1}\right)}{\log _{p}(\gamma)}$. We now equate

$$
\zeta \zeta_{\alpha} \gamma^{\log _{p}(\alpha) / \log _{p}(\gamma)}\left(\gamma^{1-k}(1+X)\right)^{\log _{p}\left(\alpha_{1}\right) / \log _{p}(\gamma)}=\zeta_{0}(1+X)^{\log _{p}\left(\alpha_{1}\right) / \log _{p}(\gamma)},
$$

where $\alpha=\zeta_{\alpha} \gamma^{\log _{p}(\alpha) / \log _{p}(\gamma)}$ for roots $\zeta_{\alpha}$ and $\zeta_{0}$ of unity. By putting $X=0$, we get

$$
\zeta \zeta_{\alpha} \gamma^{\log _{p}(\alpha) / \log _{p}(\gamma)+(1-k)\left(\log _{p}\left(\alpha_{1}\right) / \log _{p}(\gamma)\right)}=\zeta_{0},
$$

which shows that

$$
\zeta_{0}=\zeta \zeta_{\alpha} \text { and }(k-1) s_{1}=\log _{p}(\alpha) / \log _{p}(\gamma) .
$$

We conclude that $\alpha_{1}=\sqrt[k-1]{\langle\alpha\rangle}$ for $\langle\alpha\rangle=\alpha \zeta_{\alpha}^{-1}$, which is a Weil $l$-number of weight 1.

We now assume that $A \in W[[X]]\left[(1+X)^{1 / p^{n}}\right]$. Since

$$
\operatorname{Spf}\left(W[[X]]\left[(1+X)^{1 / p^{n}}\right]\right) \cong \widehat{\mathbb{G}}_{m} \stackrel{t \mapsto t^{p^{n}}}{\longrightarrow} \widehat{\mathbb{G}}_{m}=\operatorname{Spf}(W[[X]]),
$$

by applying the same argument as above to $W[[X]]\left[(1+X)^{1 / p^{n}}\right]$, we get $A(X)=$ $\zeta_{0}(1+X)^{s_{1}}$ for $s_{1}=\log _{p}\left(\alpha_{1}\right) / \log _{p}(\gamma)$, where $\alpha_{1}=\sqrt[k-1]{\langle\alpha\rangle}$ for $\langle\alpha\rangle=\alpha \zeta_{\alpha}^{-1}$.

We thus need to show that $A \in W\left[\mu_{p^{n}}\right][[X]]\left[(1+X)^{1 / p^{n}}\right]$ for sufficiently large $n$, and then the result follows from the above argument. Again we make the variable change $X \mapsto Y$ that we have already done. Replacing $A$ by $\alpha^{-1} A$ for a suitable Weil $l$-number $\alpha$ of weight $k$ (up to $\mu_{p}\left(\overline{\mathbb{Q}}_{p}\right)$ ), we may assume that there exists an infinite set $\mathcal{A}_{0} \subset \operatorname{Spec}(\mathbb{I})\left(\overline{\mathbb{Q}}_{p}\right)$ such that $P \cap \Lambda=\left(1+Y-\zeta_{P}\right)$ for $\zeta_{P} \in \mu_{p}\left(\overline{\mathbb{Q}}_{p}\right)$ and $A_{P} \in \mu_{p \infty}\left(\overline{\mathbb{Q}}_{p}\right)$ for all $P \in \mathcal{A}_{0}$. By another variable change $(1+Y) \mapsto \zeta(1+Y)$ for a suitable $\zeta \in \mu_{p}\left(\overline{\mathbb{Q}}_{p}\right)$ (as explained in the beginning of the first proof of Lemma 5.1), we may further assume that we have $P_{0} \in \mathcal{A}_{0}$ with $\zeta_{P_{0}}=1$ and $A_{P_{0}}=1$ (specifying $\alpha$ well in $\alpha \cdot \mu_{p \infty}\left(\overline{\mathbb{Q}}_{p}\right)$ ). We now write $\mathbb{J}$ for the subalgebra of $\mathbb{I}$ topologically generated by $A$ over $\Lambda=W[[Y]]$. Then we have $\mathbb{J}=\Lambda[A] \subset \mathbb{I}$. Replacing $W$ by its finite extension, we may assume that $W$ is integrally closed in $\mathbb{J}$. Since $A$ is a unit in $\mathbb{I}$, we may embed the irreducible formal scheme $\operatorname{Spf}(\mathbb{J})$ into $\widehat{\mathbb{G}}_{m} \times \widehat{\mathbb{G}}_{m}=\operatorname{Spf}\left(W\left[t, \widehat{t^{-1}, t^{\prime}}, t^{\prime-1}\right]\right)$ by the surjective $W$-algebra homomorphism $\pi: W\left[t, \widehat{t^{-1}, t^{\prime}}, t^{\prime-1}\right] \rightarrow \mathbb{J}$ sending $\left(t, t^{\prime}\right)$ to $(1+Y, A)$. Write $Z \subset \widehat{\mathbb{G}}_{m} \times \widehat{\mathbb{G}}_{m}$ for the image of $\operatorname{Spf}(\mathbb{J})$. Thus we are identifying $\Lambda$ with $\widehat{W\left[t, t^{-1}\right]}$ by $t \leftrightarrow 1+Y$. Then $P_{0} \in Z$ is the identity element of $\left(\widehat{\mathbb{G}}_{m} \times \widehat{\mathbb{G}}_{m}\right)\left(\overline{\mathbb{Q}}_{p}\right)$. Since $A$ is integral over $\Lambda$, it is a root of a monic polynomial $\Phi\left(t^{\prime}\right)=\Phi\left(t, t^{\prime}\right)=t^{\prime d}+a_{1}(t) t^{\prime d-1}+\cdots+a_{d}(t) \in \Lambda\left[t^{\prime}\right]$ irreducible over the quotient field $Q$ of $\Lambda$, and we have $\mathbb{J} \cong \Lambda\left[t^{\prime}\right] /\left(\Phi\left(t, t^{\prime}\right)\right)$. Thus $\mathbb{J}$ is free of rank $d$ over $\Lambda$; so, $\pi: Z \rightarrow \widehat{\mathbb{G}}_{m}=\operatorname{Spf}(\Lambda)$ is a finite flat morphism of degree $d$. We let $\sigma \in \operatorname{Gal}\left(\overline{\mathbb{Q}}_{p} / \mathbb{Q}_{p}\right)$ act on $\Lambda$ by $\sum_{n=0}^{\infty} a_{n} Y^{n} \mapsto \sum_{n=0}^{\infty} a_{n}^{\sigma} Y^{n}$ and on $\Lambda\left[t^{\prime}\right]$ by $\sum_{j} A_{j}(Y) t^{\prime j} \mapsto \sum_{j} A_{j}^{\sigma}(Y) t^{\prime j}$ for $A_{j}(Y) \in \Lambda$. Note that $\Phi\left(\zeta_{P}, A_{P}\right)=0$ for $P \in \mathcal{A}_{0}$. Since $A_{P} \in \mu_{p^{\infty}}\left(\overline{\mathbb{Q}}_{p}\right), A_{P}^{\sigma}=A_{P}^{\chi(\sigma)}$ for the $p$-adic cyclotomic character $\chi: \operatorname{Gal}\left(\overline{\mathbb{Q}}_{p} / \mathbb{Q}_{p}\right) \rightarrow \mathbb{Z}_{p}^{\times}$. Since $W$ is a discrete valuation ring, for its quotient field 
$F$, the image of $\chi$ on $\operatorname{Gal}\left(\overline{\mathbb{Q}}_{p} / F\right)$ is an open subgroup $U$ of $\mathbb{Z}_{p}^{\times}$. Thus we have $\Phi^{\sigma}\left(\zeta_{P}^{\chi(\sigma)}, A_{P}^{\chi(\sigma)}\right)=\Phi\left(\zeta_{P}, A_{P}\right)^{\sigma}=0$ for all $\sigma \in \operatorname{Gal}\left(\overline{\mathbb{Q}}_{p} / \mathbb{Q}_{p}\right)$ and if $\sigma \in \operatorname{Gal}\left(\overline{\mathbb{Q}}_{p} / F\right)$, $\Phi^{\sigma}=\Phi$. Thus we get

$$
\Phi\left(\zeta_{P}^{\chi(\sigma)}, A_{P}^{\chi(\sigma)}\right)=\Phi\left(\zeta_{P}, A_{P}\right)^{\sigma}=0 \text { for all } P \in \mathcal{A}_{0} .
$$

In other words, for the integral closed formal subscheme $Z_{s} \subset \widehat{\mathbb{G}}_{m} \times \widehat{\mathbb{G}}_{m}$ defined by $\Phi\left(t^{s}, t^{\prime s}\right)=0$, we have $\mathcal{A}_{0} \subset Z \cap Z_{s}$ if $s \in U$. Since $Z$ and $Z_{s}$ are finite flat over $\Lambda$ and $\mathcal{A}_{0}$ is an infinite set, we conclude that $Z=Z_{s}$. Thus $Z \subset \widehat{\mathbb{G}}_{m} \times \widehat{\mathbb{G}}_{m}$ is stable under the diagonal action $\left(t, t^{\prime}\right) \mapsto\left(t^{s}, t^{s}\right)$ for $s \in U$. We may assume that $U=1+p^{r} \mathbb{Z}_{p}$ for $r>0$. Since $Z$ is flat of relative dimension 1 over $W$, replacing $W$ by its finite extension if necessary, we find in $Z$ a $W$-point $\left(t_{0}, t_{0}^{\prime}\right) \in \widehat{\mathbb{G}}_{m}^{2}(W)$ of infinite order (indeed, $\left(t_{0}, t_{0}^{\prime}\right)=(Q, A(Q))$ for an arithmetic point $Q$ with $k(Q) \neq k$ does the job). Thus we have an infinite set $\Xi=\left\{\left(t_{0}^{s}, t^{\prime s}{ }_{0}\right) \mid s \in U\right\}=\left\{\left(t_{0} t_{0}^{p^{r} u}, t^{\prime}{ }_{0} t_{0}^{p^{r} u}\right) \mid u \in p^{n} \mathbb{Z}_{p}\right\}$ inside $Z$. By translation $\tau:\left(t, t^{\prime}\right) \mapsto\left(t t_{0}^{-1}, t^{\prime} t_{0}^{\prime-1}\right)$, we find that $\tau(Z)$ contains

$$
\Xi_{0}=\left\{\left(t_{0}^{p^{r} u}, t_{0}^{p^{r} u}\right) \mid u \in p^{n} \mathbb{Z}_{p}\right\} .
$$

Since $Z$ is integral of codimension 1 finite flat over $\operatorname{Spf}(\Lambda), \tau(Z)$ is the Zariski closure of the infinite subgroup $\Xi_{0}$ of $\widehat{\mathbb{G}}_{m}^{2}$. Then $\tau(Z)$ must be a formal subgroup of $\widehat{\mathbb{G}}_{m}^{2}$ of codimension 1 , and $Z$ is a coset $\left(t_{0}, t_{0}^{\prime}\right) \tau(Z)$ in $\widehat{\mathbb{G}}_{m} \times \widehat{\mathbb{G}}_{m}$. Since $Z$ contains the identity $P_{0}$, we must have $\tau(Z)=Z$. Since $\pi: Z \rightarrow \operatorname{Spf}(\Lambda)=\widehat{\mathbb{G}}_{m}$ is finite flat of degree $d, \pi: Z \rightarrow \widehat{\mathbb{G}}_{m}$ is an isogeny; so, $Z$ is a formal multiplicative group and is a formal subtorus of $\widehat{\mathbb{G}}_{m}^{2}$. In particular, we obtain $Z / \operatorname{Ker}\left(p^{n}: Z \rightarrow Z\right) \stackrel{\pi}{=} \widehat{\mathbb{G}}_{m}$ and hence $d=p^{n}$ for $n \geq 0$. Thus

$$
\mathbb{J}=\Lambda[A] \subset W\left[\mu_{p^{n}}\right][[Y]]\left[(1+Y)^{p^{-n}}\right]=W\left[\mu_{p^{n}}\right][[X]]\left[(1+X)^{p^{-n}}\right],
$$

as desired. This finishes the proof.

Corollary 5.3. If $a\left(p, f_{P}\right)$ for an arithmetic point $P$ with $k(P)=2$ is a root of unity (that is, the automorphic representation generated by $f_{P}$ is Steinberg at $p$ ), then the extensions $M_{H, \mathcal{A}}(\mathcal{F})$ and $\mathbb{Q}_{H, k}(\mathcal{F})$ are infinite extensions of $\mathbb{Q}\left(\mu_{p} \infty\right)$ for any integer $k \geq 2$.

Proof. Since $\mathbb{Q}_{H, k}(\mathcal{F}) \supset M_{H, \mathcal{A}}(\mathcal{F})$, we prove infiniteness of $\left[M_{H, \mathcal{A}}(\mathcal{F}): \mathbb{Q}\left(\mu_{p^{\infty}}\right)\right]$. If $M_{H, \mathcal{A}}(\mathcal{F})$ is a finite extension of $\mathbb{Q}\left(\mu_{p}\right)$, we are in Case (1) of the above proposition for $l=p$. Then $a\left(p, f_{P}\right)$ for a weight 2 arithmetic point $P$ is a weight 1 Weil $p$ number that is not a root of unity. Thus if we have one arithmetic point with a root of unity $a\left(p, f_{P}\right)$, then $M_{H, \mathcal{A}}(\mathcal{F})$ has to be an infinite extension of $\mathbb{Q}\left(\mu_{p^{\infty}}\right)$.

\section{Proof of the strong horizontal theorem}

Let $X$ be the variable of $W[[\Gamma]]$ centered at weight 1 . We start with a couple of preliminary results. Consider the endomorphism $\sigma_{s}:(1+X) \mapsto(1+X)^{s}=$ $\sum_{n=0}^{\infty}\left(\begin{array}{l}s \\ n\end{array}\right) X^{n}$ of a power series ring $B[[X]]$ over $B$. Take $B=W$ and write $\sigma=\sigma_{2}$. Let $G$ be a group.

Lemma 6.1. Let the notation be as above. Let $A$ be an integral domain over $W[[X]]$ of characteristic different from 2 with quotient field $F$. Assume that the endomorphism $\sigma_{2}$ on $W[[X]]$ extends to an endomorphism $\sigma$ of the field $F$. Let $\rho: G \rightarrow G L_{2}(F)$ be a representation, and put $\rho^{\sigma}:=\sigma \circ \rho$. Let $H \subset G$ be a 
subgroup of $G$, and put $\rho_{H}:=\left.\rho\right|_{H}$. Suppose we have a subgroup $H$ of $G$ such that $\operatorname{Tr}\left(\rho_{H}^{\sigma}\right)=\operatorname{Tr}\left(\rho_{H}^{2}\right)$. Then $\rho_{H}$ is not absolutely irreducible.

Here $\rho_{H}^{2}: H \rightarrow G L_{2}(F)$ is the map sending $h$ to the square of $\rho_{H}(h)$.

Proof. We suppose that $\rho_{H}$ is absolutely irreducible over $F$, and we try to get absurdity. We have the identity $\operatorname{Tr}\left(\rho_{H}^{\sigma}\right)=\operatorname{Tr}\left(\rho_{H}^{2}\right)=\operatorname{Tr}\left(\rho_{H}^{\text {sym } \otimes 2}\right)-\operatorname{det}\left(\rho_{H}\right)$ for the symmetric second tensor representation $\rho_{H}^{\text {sym } \otimes 2}$ of $\rho_{H}$. Over $F$, by absolute irreducibility, we have the identity of semi-simplification: $\left(\rho_{H}^{s y m \otimes 2}\right)^{s s} \cong \rho_{H}^{\sigma} \oplus \operatorname{det}\left(\rho_{H}\right)$. Tensoring $\operatorname{det}(\rho)^{-1}$ and writing $A d\left(\rho_{H}\right)$ for $\rho_{H}^{\text {sym } \otimes 2} \otimes \operatorname{det}\left(\rho_{H}\right)^{-1}$, we get

$$
A d\left(\rho_{H}\right)^{s s} \cong\left(\rho_{H}^{\sigma} \otimes \operatorname{det}(\rho)^{-1}\right) \oplus \mathbf{1} \text {. }
$$

The representation $A d\left(\rho_{H}\right)$ can be realized on the space

$$
V:=\left\{x \in \operatorname{End}_{F}\left(F^{2}\right) \mid \operatorname{Tr}(x)=0\right\}
$$

with the action $\operatorname{Ad}\left(\rho_{H}\right)(h) x=\rho_{H}(h) x \rho_{H}(h)^{-1}$. Since $\rho_{H}^{\sigma}$ is absolutely irreducible, we have either 1 as a subrepresentation of $\operatorname{Ad}\left(\rho_{H}\right)$ or a quotient of $\operatorname{Ad}\left(\rho_{H}\right)$. Since $A d\left(\rho_{H}\right)$ is self-dual under the trace pairing $\langle x, y\rangle=\operatorname{Tr}(x y)$ for $x, y \in V$ (as $A$ is not of characteristic 2), in the latter case of having $\mathbf{1}$ as a quotient, by duality, $\mathbf{1}$ is also a subrepresentation of $A d\left(\rho_{H}\right)$. Thus we have $\mathbf{1} \hookrightarrow A d\left(\rho_{H}\right)$ as $H$-modules. In other words, we have a nontrivial element $0 \neq \phi \in \operatorname{End}_{A[H]}\left(\rho_{H}\right)$ such that $\operatorname{Tr}(\phi)=0$. Since $\rho_{H}$ is absolutely irreducible, $\phi$ has to be a scalar multiplication by $z \in A^{\times}$ by Schur's lemma; so, $\operatorname{Tr}(\phi)=2 z \neq 0$, a contradiction (unless $A$ has characteristic 2).

Suppose $p>2$. Let $Q$ be the quotient field of $W[[X]]$; so, $\sigma:=\sigma_{2}$ extends to a field automorphism of $Q$ uniquely, which we denote again by $\sigma$. Then by the Steinitz theorem (e.g., BAL, V.4.2]), $\sigma$ extends to an automorphism of an algebraic closure $\bar{Q}$ of $Q$. Let $G:=\operatorname{Gal}(\overline{\mathbb{Q}} / \mathbb{Q})$. Taking $A=\bar{Q}$ and embedding $\mathbb{I}$ into $\bar{Q}$ as the quotient field of $\mathbb{I}$ is a finite extension of $Q$, we can apply the above lemma to $\rho_{\mathbb{I}}$. Now we want to make a choice of the subgroup $H$. Let $I$ be an open ideal of the profinite ring $\mathbb{I}$ and put

$$
H=H_{I}=\left\{h \in G \mid \operatorname{Tr}\left(\rho_{\mathbb{I}}(h \tau)\right) \equiv \operatorname{Tr}\left(\rho_{\mathbb{I}}(\tau)\right) \equiv \operatorname{Tr}\left(\rho_{\mathbb{I}}(\tau h)\right) \quad \bmod I \text { for all } \tau \in G\right\} .
$$

This group is the stabilizer of the trace map modulo $I$ (i.e., the kernel of the pseudorepresentation associated to $\operatorname{Tr}(\rho)$; see $[\mathrm{MFG}, \S 2.2])$; so, it is a normal subgroup of $\operatorname{Gal}(\overline{\mathbb{Q}} / \mathbb{Q})$, and $\operatorname{Tr}(h) \bmod I$ for $h \in H$ only depends on the coset $h H=H h$. Since $I$ is an open ideal of $\mathbb{I}, \operatorname{Tr}\left(\rho_{\mathbb{I}}\right) \bmod I$ has values in the finite $\operatorname{ring} \mathbb{I} / I$; so, its stabilizer $H_{I}$ is an open subgroup of $\operatorname{Gal}(\overline{\mathbb{Q}} / \mathbb{Q})$. If $\rho_{\mathbb{I}}$ has values in $G L_{2}(\mathbb{I})$ with absolutely irreducible $\rho_{\mathbb{I}} \bmod \mathfrak{m}_{\mathbb{I}}$, then $H_{I}$ is equal to $\operatorname{Ker}\left(\rho_{\mathbb{I}} \bmod I\right)$ (by the theory of pseudo-representation; see MFG, $\S 2.1 .7$ and $\S 2.2 .1])$. By the above trace identity: $\operatorname{Tr}\left(\rho_{\mathbb{I}}(h \tau)\right) \equiv \operatorname{Tr}\left(\rho_{\mathbb{I}}(\tau)\right) \equiv \operatorname{Tr}\left(\rho_{\mathbb{I}}(\tau h)\right) \bmod I$, taking $\tau=h^{n}$, we get $\operatorname{Tr}\left(\rho_{\mathbb{I}}\left(h^{n+1}\right)\right) \equiv \operatorname{Tr}\left(\rho_{\mathbb{I}}\left(h^{n}\right)\right) \bmod I$ for all $n \in \mathbb{Z}$, which implies $\operatorname{Tr}\left(\rho_{\mathbb{I}}\left(h^{n}\right)\right) \equiv 2$ $\bmod I$ (for any $n \in \mathbb{Z}$ ) if $h \in H_{I}$. Since $2 \cdot \operatorname{det}\left(\rho_{\mathbb{I}}(h)\right)=\operatorname{Tr}\left(\rho_{\mathbb{I}}\left(h^{2}\right)\right)-\operatorname{Tr}\left(\rho_{\mathbb{I}}(h)\right)^{2}$, as $p>2$, we have $\operatorname{det}\left(T-\rho_{\mathbb{I}}(h)\right) \equiv(T-1)^{2} \bmod I$ if $h \in H_{I}$; so, the eigenvalues $\alpha$ of $\rho_{\mathbb{I}}(h)$ satisfy $(\alpha-1)^{2} \equiv 0 \bmod I$ as long as $h \in H_{I}$ and $p \neq 2$. We write $\rho_{I}=\left.\rho_{\mathbb{I}}\right|_{H_{I}}$.

Lemma 6.2. Let the notation be as above. Let $\mathcal{F}$ be a p-adic analytic family of slope 0 with coefficients in $\mathbb{I}$ with Galois representation $\rho:=\rho_{\mathbb{I}}: \operatorname{Gal}(\overline{\mathbb{Q}} / \mathbb{Q}) \rightarrow G L_{2}(\bar{Q})$. Write $\sigma$ for an extension of $\sigma_{2}$ on $W[[X]]$ to the algebraic closure of the field of 
fractions of $\mathbb{I}$. If $\operatorname{Tr}\left(\rho_{I}^{\sigma}\right)=\operatorname{Tr}\left(\rho_{I}^{2}\right)$ for an open ideal $I \subsetneq \mathbb{I}$, then there exists an imaginary quadratic extension $M / \mathbb{Q}$ and a character $\varphi: \operatorname{Gal}(\overline{\mathbb{Q}} / M) \rightarrow \mathbb{I}^{\times}$such that $\rho \cong \operatorname{Ind}_{M}^{\mathbb{Q}} \varphi$ over $\bar{Q}$.

Proof. By Lemma 6.1 applied to $H=H_{I}$ and $\rho_{I}=\left.\rho\right|_{H_{I}}, \rho_{I}$ is reducible. Write $\rho_{I}=\left(\begin{array}{cc}\epsilon & * \\ 0 & \delta\end{array}\right)$, extending the scalar $\mathbb{I}$ if necessary. Since $\rho$ extends $\rho_{I}, g \mapsto \rho_{I}^{h}(g):=$ $\rho_{I}\left(h g h^{-1}\right)=\rho(h) \rho_{I}(g) \rho(h)^{-1}$ is equivalent to $\rho_{I}$ for all $h \in \operatorname{Gal}(\overline{\mathbb{Q}} / \mathbb{Q})$. Thus $G:=\operatorname{Gal}(\overline{\mathbb{Q}} / \mathbb{Q})$ acts on $\{\delta, \epsilon\}$ by inner conjugation:

$$
\left(\begin{array}{cc}
\epsilon^{h} & * \\
0 & \delta^{h}
\end{array}\right)=\rho(h)\left(\begin{array}{cc}
\epsilon & * \\
0 & \delta
\end{array}\right) \rho(h)^{-1} .
$$

Let $\Delta \subset G$ be the stabilizer of $\delta$. Then $M=\overline{\mathbb{Q}}^{\Delta}$ is at most a quadratic extension of $\mathbb{Q}$. If $\delta \neq \epsilon$ or $\rho_{I}$ is nonsemisimple, by (6.1), $\rho_{\Delta}=\left.\rho\right|_{\Delta}$ has to be upper triangular. Thus the two characters extend to $\delta, \epsilon: \Delta \rightarrow \mathbb{I}^{\times}$, and $\rho_{\Delta}^{s s}=\delta \oplus \epsilon$. Since $\rho$ is absolutely irreducible (over the quotient field $Q$ of $\mathbb{I}$ ), $[G: \Delta]=2$ and by Frobenius reciprocity, $\rho \cong \operatorname{Ind}_{M}^{\mathbb{Q}} \delta \cong \operatorname{Ind}_{M}^{\mathbb{Q}} \epsilon$. As already remarked in (CM1) in Section 1. this can happen only for imaginary $M$ for Galois representations associated to a $p$-adic analytic family of modular forms (of slope 0 ).

Suppose that $\delta=\epsilon$. Let $D$ be the decomposition group at $p$. Then $\rho_{D}:=\left.\rho\right|_{D}$ is reducible with two distinct diagonal characters (by (Gal)): one trivial and another $\varepsilon$ giving the $W[[X]]$-algebra structure (centered at weight 1 ) of $\mathbb{I}$ (see (Gal)). In particular, $\varepsilon$ is of infinite order. Thus we may assume that $\delta$ restricted to $D$ is trivial and $\epsilon$ restricted to $D$ has infinite order agreeing with $\varepsilon$. Then it is impossible to have $\delta=\epsilon$, because $\mathbb{I} / I$ is a finite ring.

Proof of Theorem 3.3, Let $K:=\mathbb{Q}\left(\mu_{p \infty}\right)$ and $L_{P}=K\left(\alpha_{l, P}\right)$ for a prime $l$. By Corollary 4.2, we need to prove that $\left[M_{H, \mathcal{A}}(\mathcal{F}): K\right]<\infty \Rightarrow \mathcal{F}$ has CM. Suppose $\left[M_{H, \mathcal{A}}(\mathcal{F}): K\right]<\infty$. For each arithmetic $P$ with $k(P)=k$, by Lemma 4.3 ,

$$
\left[K\left(f_{P}\right): K\left(a\left(p, f_{P}\right)\right)\right]<d
$$

for a positive integer $d$ independent of $P$. Thus $\left[L_{P}: K\right]<2 d\left[M_{H, \mathcal{A}}(\mathcal{F}): K\right]$ for each prime $l$. Therefore, any odd prime $l>2 d\left[M_{H, \mathcal{A}}(\mathcal{F}): K\right]$ is at most tamely ramified in $L_{P} / K$. Take such an odd prime $l>2 d\left[M_{H, \mathcal{A}}(\mathcal{F}): K\right]$ prime to $N p$. Let $\rho=\rho_{\mathbb{I}}$ be the Galois representation associated to $\mathcal{F}$. Thus by Proposition [5.2, we have $\operatorname{Tr}\left(\rho\left(F_{\text {rob }}\right)\right)=\zeta(1+X)^{a}+\zeta^{\prime}(1+X)^{a^{\prime}}$ for two roots of unity $\zeta, \zeta^{\prime}$ and $a, a^{\prime} \in \mathbb{Q}_{p}$ with denominator bounded independent of $l$ (i.e., $a p^{B} \in \mathbb{Z}_{p}$ and $a^{\prime} p^{B} \in \mathbb{Z}_{p}$ for an integer $B \geq 0$ independent of $l$ ).

Take an arithmetic $P_{0} \in \operatorname{Spec}(\mathbb{I})\left(\overline{\mathbb{Q}}_{p}\right)$ to see that the order of $\zeta$ is bounded independent of $l$. Let $\alpha$ be a root of $\operatorname{det}\left(X-\rho_{P_{0}}\left(F r o b_{l}\right)\right)=0$ in $\overline{\mathbb{Q}}_{p}$. Then $\left[\mathbb{Q}\left(f_{P_{0}}, \alpha\right): \mathbb{Q}\left(f_{P_{0}}\right)\right] \leq 2$. Write $m=\left[\mathbb{Q}\left(f_{P_{0}}\right): \mathbb{Q}\right], \zeta=\zeta_{p} \zeta^{(p)}$ with $\zeta_{p} \in \mu_{p}$ and $\zeta^{(p)}$ of order prime to $p$. Since $(1+X)^{s} \equiv 1 \bmod \mathfrak{m}_{\mathbb{I}}$, the order of $\zeta^{(p)}$ is bounded by the degree of the residue fields modulo $p$ of the integer ring of $\mathbb{Q}_{p}\left(f_{P_{0}}, \alpha\right)$. Thus the order of $\zeta^{(p)}$ is bounded by $p^{2 m}$. Note that $P_{0}\left((1+X)^{a}\right)=(1+X)^{a} \bmod P_{0}$ is in a finite extension $L$ of $\mathbb{Q}_{p}$ depending only on the denominator $p^{n}$ of $a$. For example, if $P_{0}$ contains $(1+X)-\gamma^{k}, L \subset \mathbb{Q}_{p}\left[\gamma^{a}\right]=\mathbb{Q}_{p}\left[\sqrt[p^{n}]{(1+p)}\right] \subset \mathbb{Q}_{p}\left[\sqrt[p^{B}]{(1+p)}\right]$ for $\gamma=1+p$. We have $\zeta_{p} \in L\left[\zeta_{p}\right]$ whose degree is bounded by $2 m\left[L: \mathbb{Q}_{p}\right]$; so, the order of $\zeta_{p}$ is also bounded independent of $l$. Replacing $W$ by its finite extension, we may assume that all such roots of unity are in $W$. 
Let $I=\mathfrak{m}_{\mathbb{I}}^{2 N}+(X)$ for a sufficiently large $N$ and $F$ be the fixed field of $H_{I}$. Then $\mathbb{I} / I \cong W / \mathfrak{m}_{W}^{2 N}$. We have $\operatorname{Tr}\left(\rho\left(F r o b_{\mathfrak{l}}\right)\right)=\zeta^{f}(1+X)^{f a}+{\zeta^{\prime}}^{f}(1+X)^{f a^{\prime}} \equiv \zeta^{f}+\zeta^{\prime f}$ $\bmod I$, and $\operatorname{det}\left(T-\rho\left(\right.\right.$ Frob $\left.\left._{\mathfrak{l}}\right)\right) \equiv(T-1)^{2} \bmod I$ for a prime $\mathfrak{l} \mid l$ of $F$ of residual degree $f$. So $\left(\zeta^{f}-1\right)^{2} \equiv 0 \bmod \mathfrak{m}_{W}^{2 N}$. Since any square 0 element in $W / \mathfrak{m}_{W}^{2 N}$ is contained in $\mathfrak{m}_{W}^{N} / \mathfrak{m}_{W}^{2 N}$, we have $\zeta^{f} \equiv 1 \bmod \mathfrak{m}_{W}^{N}$; so, taking $N$ large, we may assume that $\zeta^{f}={\zeta^{\prime}}^{f}=1$. This shows that $\operatorname{Tr}\left(\sigma_{s}\left(\rho\left(F_{r o b}\right)\right)\right)=\operatorname{Tr}\left(\rho\left(F_{l} o b_{l}\right)^{s}\right)$ for all $0 \neq s \in \mathbb{Z}_{p}$. Thus by the Chebotarev density theorem, we get $\operatorname{Tr}\left(\sigma_{s}\left(\rho_{I}\right)\right)=\operatorname{Tr}\left(\rho_{I}^{s}\right)$ over $\operatorname{Gal}(\overline{\mathbb{Q}} / F)$. In particular, we have $\operatorname{Tr}\left(\sigma_{2}\left(\rho_{I}\right)\right)=\operatorname{Tr}\left(\rho_{I}^{2}\right)$. Then by Lemma 6.2 . $\rho=\operatorname{Ind}_{M}^{\mathbb{Q}} \varphi$ for an imaginary quadratic field $M$. Thus $\mathcal{F}$ has to be a CM family.

Here is an obvious corollary of the above proof.

Corollary 6.3. Let $K:=\mathbb{Q}\left[\mu_{p} \infty\right]$ and $\mathcal{A} \subset \operatorname{Spec}(\mathbb{I})\left(\mathbb{C}_{p}\right)$ be an infinite set of arithmetic points $P$ with fixed weight $k(P)=k \geq 2$. Unless $\mathcal{F}$ has complex multiplication, $\lim \sup _{P \in \mathcal{A}}\left[K\left(a\left(p, f_{P}\right)\right): K\right]=\infty$.

Indeed, if $\lim \sup _{P}\left[K\left(a\left(p, f_{P}\right)\right): K\right]<\infty$, the index $\left[L_{P}: K\right](P \in \mathcal{A})$ is bounded for $A \in \mathbb{I}$ as in Proposition 5.2 . We can still apply the above proof and conclude that $\mathcal{F}$ has complex multiplication. We also conclude that $\lim _{\sup }\left[K\left(f_{P}\right): K\right]=\infty$ as in the horizontal theorem in the introduction.

\section{Results towards Vertical CONJECTURES}

We start with

Lemma 7.1. Let $f \in S_{2}\left(\Gamma_{0}\left(N p^{r+1}\right), \psi\right)$ ( $\left.p \nmid N\right)$ be a p-ordinary Hecke eigenform with exact level $N p^{r+1}$ for $r \geq 0$. Then, $f$ is old at $p$ if and only if $r=0$, $\left.\psi\right|_{(\mathbb{Z} / p \mathbb{Z}) \times}=1$ and $f$ is associated to a Hecke eigenform of level $N$. Suppose either that the p-component $\psi_{p}:=\left.\psi\right|_{\mathbb{Z}_{p}^{\times}}$is nontrivial or that $f$ is old at $p$. Then $\mathbb{Q}(f)$ is a $C M$ field, and $a(p, f)$ is a Weil p-number of weight 1 . Let $R$ (resp. $R^{+}$) be the integer ring of $\mathbb{Q}(a(p, f))$ (resp. the maximal real subfield of $\mathbb{Q}(a(p, f)))$. If either $p$ is unramified in $\mathbb{Q}(a(p, f)) / \mathbb{Q}$ or $a(p, f)$ generates $R_{p}$ over $R_{p}^{+}$, there exists

a p-ordinary CM-type $\Sigma_{p}=\{\mathfrak{P} \mid p\}$ of $\mathbb{Q}(f)$ such that $(a(p, f))=\prod_{\mathfrak{P} \in \Sigma_{p}^{c}} \mathfrak{P}^{e(\mathfrak{P})}$ for the ramification index $e(\mathfrak{P})$ of $\mathfrak{P} /(p)$.

Proof. Since $f$ is ordinary at $p$, the $p$-component $\pi_{p}$ of the automorphic representation generated by $f$ is either in principal series $\pi(\alpha, \beta)$ or a Steinberg representation $\sigma\left(|\cdot|_{p}^{-1} \alpha, \alpha\right)$ for an unramified character $\alpha$ of $\mathbb{Q}_{p}^{\times}$(with $\alpha(p)=a(p, f)$ ). As is well known, $f$ is old if and only if $\beta$ is unramified (so, $f$ comes from a primitive new form of prime-to- $p$ level). By the congruence relation (proved by Eichler, Shimura, Igusa and Deligne/Rapoport; see [AT] and [GME, Section 4.2]), $a(p, f)$ is a Weil $p$ number of weight 1 . Then the rest of the assertions follow from Proposition 2.1.

Write $M_{V, r}(\mathcal{F})=M_{V, \mathcal{A}_{r}}(\mathcal{F})$ for the set $\mathcal{A}_{r}$ of all arithmetic points of $\operatorname{Spec}(\mathbb{I})\left(\mathbb{C}_{p}\right)$ with $r(P) \leq r$.

Proposition 7.2. Let $\mathcal{F}=\left\{f_{P}\right\}_{P \in \operatorname{Spec}(\mathbb{I})\left(\overline{\mathbb{Q}}_{p}\right)}$ be a p-adic analytic family of classical p-ordinary Hecke eigenforms and $\mathcal{A} \subset \operatorname{Spec}(\mathbb{I})\left(\overline{\mathbb{Q}}_{p}\right)$ be an infinite set of arithmetic points $P$ with $r(P) \leq r$ for a fixed $r \geq 0$ such that $M_{V, \mathcal{A}}(\mathcal{F})$ is a finite extension over $\mathbb{Q}$. Assume that for $P_{0} \in \mathcal{A}$,

(1) $\alpha_{0}=a_{P_{0}}(p)$ is a Weil p-number of weight $k\left(P_{0}\right)-1$, 
(2) $\Sigma_{\alpha_{0}}=\left\{\sigma: \mathbb{Q}\left(\alpha_{0}\right) \hookrightarrow \overline{\mathbb{Q}}|| i_{p}\left(\alpha_{0}^{\sigma}\right) \mid=1\right\}$ is a CM type of $\mathbb{Q}\left(\alpha_{0}\right)$.

Then there exist a Weil p-number $\alpha$ of weight 1 with $\left|i_{p}(\alpha)\right|_{p}=1$ such that $a(p)_{P}=$ $\zeta\langle\alpha\rangle^{k(P)-1}$ for a root of unity $\zeta$ for all arithmetic $P$ with $k(P) \geq 2$, where $\langle\alpha\rangle=$ $\exp _{p}\left(\log _{p}\left(i_{p}(\alpha)\right)\right)$ for the Iwasawa p-adic logarithm $\log _{p}$. In particular, $M_{V, r}(\mathcal{F})$ is a finite $C M$ extension of $M_{V, \mathcal{A}}(\mathcal{F})$.

Proof. In order to give a simple sketch of the proof, suppose first that $M:=$ $M_{V, \mathcal{A}}(\mathcal{F})$ is an imaginary quadratic field. Thus $\Sigma_{\alpha_{0}}$ is made of a single element $\iota=\left.i_{p}\right|_{M}$, and for each $k$, there exists at most one Weil number $\alpha_{k} \in M$ of weight $k$ (up to roots of unity in $M$ ) such that $\left|\alpha_{k}\right|_{p}=1$. In $M,\left(\alpha_{k}\right)=\overline{\mathfrak{p}}^{k}$ for the prime ideal $\mathfrak{p}$ of $M$ corresponding to $\left.i_{p}\right|_{M}$. Fix such a $k$. Taking a $k$-th root $\alpha=\sqrt[k]{\alpha_{k}}$, we have $\alpha_{l}=\alpha^{l}$ up to roots of unity for all $l$ as $\left(\alpha_{l}\right)=\overline{\mathfrak{p}}^{l}$.

Since $\mathcal{A}$ is an infinite set, there exists an infinite sequence $P_{1}, P_{2}, \ldots, P_{n}, \ldots$ in $\mathcal{A}$ with increasing weight $k\left(P_{1}\right)<k\left(P_{2}\right)<\cdots$ such that $\left(a_{P_{j}}(p)\right)=\overline{\mathfrak{p}}^{k\left(P_{j}\right)-1}$ for all $j>0$. Put

$$
\langle\alpha\rangle=\exp _{p}\left(\frac{1}{k\left(P_{0}\right)-1} \log _{p}\left(a\left(p, f_{P_{0}}\right)\right)\right)=\exp _{p}\left(\log _{p}(\alpha)\right) .
$$

Since $\left(a_{P_{j}}(p)\right)=\overline{\mathfrak{p}}^{\left(k\left(P_{j}\right)-1\right)}, a_{P_{j}}(p) /\langle\alpha\rangle^{k\left(P_{j}\right)-1}$ is a Weil number of weight 0 ; that is, it is an algebraic integer with all its conjugates having absolute value 1 . Then by Kronecker's theorem, we find $a_{P_{j}}(p)=\zeta_{P_{j}}\langle\alpha\rangle^{k\left(P_{j}\right)-1}$ for a root of unity $\zeta_{P_{j}}$. Note that $\langle\alpha\rangle$ is contained in a finite extension $M^{\prime} / M$. Since there are finitely many roots of unity in $M^{\prime}$, we have only finitely many possibilities for $\zeta_{P_{j}}$. Therefore, replacing $\left\{P_{j}\right\}_{j}$ by its subsequence, we find an infinite sequence $P_{1}, P_{2}, \ldots, P_{n}, \ldots$ of increasing weights such that $a_{P_{j}}(p)=\zeta\langle\alpha\rangle^{k\left(P_{j}\right)-1}$ for all $j=1,2, \ldots$ for a fixed root of unity $\zeta$. We have a power series $\Phi_{\alpha}(X) \in W[[X]]\left[(1+X)^{1 / p^{n}}\right]$ (for a suitable $n \geq 0$ ) with coefficients in a discrete valuation ring $W$ finite flat over $\mathbb{Z}_{p}$ such that $\Phi_{\alpha}\left(\gamma^{k}-1\right)=\zeta\langle\alpha\rangle^{k}$ for all integers $k$. Indeed, $\Phi_{\alpha}(X)=\zeta(1+X)^{s}$ for $s=\log _{p}(\alpha) / \log _{p}(\gamma)$. Since $M$ is an imaginary quadratic field in which $p$ splits into $(p)=\mathfrak{p} \overline{\mathfrak{p}}$, we have $M_{\mathfrak{p}}=\mathbb{Q}_{p}$ and hence $\log _{p}(\alpha)=\frac{\log \left(a_{P_{0}}(p)\right)}{k\left(P_{0}\right)-1} \in M_{\mathfrak{p}}=\mathbb{Q}_{p}$. Thus $s \in \mathbb{Q}_{p}$, and $p^{n}$ is the denominator of $s$. Since $\mathcal{F}$ is an ordinary family, there exists an element $A \in \mathbb{I}$ such that $a\left(p, f_{P}\right)=(A \bmod P)$ for all height 1 primes $P$ of $\mathbb{I}$ containing $\left(1+X-\gamma^{k(P)}\right)$. Thus we find $A \equiv \Phi_{\alpha} \bmod P_{j}$ for infinitely many distinct primes $P_{j}$; so, $A=\Phi_{\alpha}$, as desired.

We now treat the general case where $M$ may not be an imaginary quadratic field. Let $K \subset \overline{\mathbb{Q}}$ be a number field with integer ring $O$. Consider $O \otimes_{\mathbb{Z}} K$. Then $O \otimes_{\mathbb{Z}} K$ is a product of fields $\sigma(O) K \subset \overline{\mathbb{Q}}$ indexed by a set of embeddings $S:=\{\sigma: O \hookrightarrow \overline{\mathbb{Q}}\}$. Take the base ring $W$ containing $O$. Then $\mathbb{I} \otimes_{\mathbb{Z}} K$ contains $O \otimes_{\mathbb{Z}} K$, and $\mathbb{I} \otimes_{\mathbb{Z}} K$ decomposes accordingly: $\mathbb{I} \otimes_{\mathbb{Z}} K=\prod_{\sigma \in S} \mathbb{I}_{\sigma}$. Regard $\mathbb{I} \otimes_{\mathbb{Z}} K$ as a $K$-algebra from the right factor (and $K$ is embedded in $\overline{\mathbb{Q}}_{p}$ by $i_{p}$ ). Note that $\mathbb{I} \otimes_{\mathbb{Z}} K=\mathbb{I} \otimes_{\mathbb{Z}_{p}} \mathbb{Z}_{p} \otimes_{\mathbb{Z}} K=\mathbb{I} \otimes_{\mathbb{Z}_{p}} K_{p}$ for $K_{p}=\mathbb{Z}_{p} \otimes_{\mathbb{Z}} K$. For an arithmetic prime $P$, we have $\mathbb{Z}\left[f_{P}\right]:=\mathbb{Z}\left[a_{P}(n) \mid n=1,2, \ldots\right] \subset \mathbb{I} / P$. Then $\mathbb{Z}\left[f_{P}\right] \otimes_{\mathbb{Z}} K \subset \mathbb{I} / P \otimes_{\mathbb{Z}} K$ as $K$ is $\mathbb{Z}$-flat. On the other hand,

$$
\mathbb{Z}\left[f_{P}\right] \otimes_{\mathbb{Z}} K=\mathbb{Q}\left(f_{P}\right) \otimes_{\mathbb{Z}} K \cong \prod_{\tau: \mathbb{Q}\left(f_{P}\right) \hookrightarrow \overline{\mathbb{Q}}_{p}} i_{p}\left(\tau\left(\mathbb{Q}\left(f_{P}\right)\right) K\right) .
$$

The composite $\tau\left(\mathbb{Q}\left(f_{P}\right)\right) K$ is taken in $\overline{\mathbb{Q}}_{p}$ by sending it by $i_{p}$ inside $\overline{\mathbb{Q}}_{p}$. For some $\tau$ (for example, complex conjugation $\tau=c$ ), we may have $\left|i_{p}\left(\tau\left(a_{P}(p)\right)\right)\right|_{p}<1$. 
Let us give more details why this strange phenomenon: $\left|i_{p}\left(\tau\left(a_{P}(p)\right)\right)\right|_{p}<1$ could occur. Suppose $K / \mathbb{Q}$ is a Galois extension with $O \subset W$. Then writing $V=K \cap W$ (the valuation ring corresponding to $i_{p}: K \hookrightarrow \overline{\mathbb{Q}}_{p}$ ), we have

$$
V \otimes_{\mathbb{Z}} V \subset \prod_{\sigma \in \operatorname{Gal}(K / \mathbb{Q})} \sigma(V) V .
$$

Let $e_{\sigma}$ for the idempotent of $\sigma(V) V$. Writing $D_{V} \subset \operatorname{Gal}(K / \mathbb{Q})$ for the decomposition subgroup of $V$, unless $\sigma \in D_{V}$ (i.e., $\left.\sigma(V)=V\right), \sigma(V) V=K$. Since $V$ is embedded in $\mathbf{h}_{k(P), \psi_{P}}$, we regard $e_{\sigma} \in \mathbf{h}_{k(P), \psi_{P}} \otimes_{\mathbb{Z}} V$. Since $U(p)$ is invertible in $\mathbf{h}_{k(P), \psi_{P}}$, the image of $e_{\sigma}(U(p) \otimes 1)$ is invertible in $K=\sigma(V) V$, but that does not mean that $e_{\sigma}(U(p) \otimes 1)$ is a $p$-adic unit. Define

$$
E_{P}=\lim _{n \rightarrow \infty}(U(p) \otimes 1)^{n !}
$$

under the $p$-adic topology $\mathcal{T}_{P}$ of $\mathbf{h}_{k(P), \psi_{P}} \otimes_{\mathbb{Z}} V$ inducing the natural topology on $1 \otimes V \subset \mathbf{h}_{k(P), \psi_{P}} \otimes_{\mathbb{Z}} V$. Then $E_{P}$ is orthogonal to $e_{\sigma}$ if $e_{\sigma}(U(p) \otimes 1)$ is p-adically nilpotent under the $p$-adic topology $\mathcal{T}_{P}$ of $\mathbf{h}_{k(P), \psi_{P}} \otimes_{\mathbb{Z}} V$. The idempotent $e_{P}=$ $\lim _{n \rightarrow \infty} U(p)^{n !}$ in $h_{k(P), \psi_{P}}$ (for $\psi_{P}=\psi_{k(P)} \varepsilon_{P}$ ) is only defined over $\overline{\mathbb{Q}}$; so, $e_{P}$ may not commute with some $\sigma$. In other words, we could have $e_{P} \otimes 1 \neq E_{P}$, and

$$
E_{P}=\sum_{\tau:\left|i_{p}\left(\tau\left(a_{P}(p)\right)\right)\right|_{p}=1} e_{\tau} .
$$

We can embed $\mathbf{h}$ into $\prod_{P \in \mathcal{A}} \mathbf{h}_{k(P), \psi_{P}} \subset \prod_{P \in \mathcal{A}} h_{k(P), \psi_{P}}$ for any infinite set $\mathcal{A}$ of arithmetic points $P$ of $W[[T]]$ sending $T(n)$ to diagonal $T(n)$ in the product of the right-hand side. The tensor product $\mathbf{h} \otimes_{\mathbb{Z}} K$ is embedded in $\prod_{P}\left(h_{k(P), \psi_{P}} \otimes_{\mathbb{Z}} K\right)$. We write $E=\prod_{P} E_{P}$, which is an idempotent of $\prod_{P}\left(h_{k(P), \psi_{P}} \otimes_{\mathbb{Z}} K\right)$ but may not be in $\left(\prod_{P} h_{k(P), \psi_{P}}\right) \otimes_{\mathbb{Z}} K$. The closure $\widehat{\mathbf{h} \otimes_{\mathbb{Z}} K}$ of $\mathbf{h} \otimes_{\mathbb{Z}} K$ inside $\prod_{P}\left(h_{k(P), \psi_{P}} \otimes_{\mathbb{Z}} K\right)$ contains $E$ (as $\left.E=\lim _{n \rightarrow \infty} U(p)^{n !} \otimes 1\right)$, and $E\left(\widehat{\mathbf{h} \otimes_{\mathbb{Z}} K}\right)$ is free of finite rank over $W[[T]]\left[\frac{1}{p}\right]$ (though $\widehat{\mathbf{h} \otimes_{\mathbb{Z}} K}$ could be huge). Each irreducible component of $E\left(\widehat{\mathbf{h} \otimes_{\mathbb{Z}} K}\right)$ gives rise to another $p$-adic analytic family of slope 0 .

Pick an arithmetic point $P$, and write $\alpha=a_{P}(p)$. Take an irreducible component $\operatorname{Spec}\left(\mathbb{I}_{\sigma}^{\circ}\right)$ of $\operatorname{Spec}\left(\mathbb{I}_{\sigma}\right) \cap \operatorname{Spec}\left(E\left(\widehat{\mathbf{h} \otimes_{\mathbb{Z}} K}\right)\right)$. Let $P_{\tau}$ be a factor of $P \otimes_{\mathbb{Z}} K \subset \mathbb{I} \otimes_{\mathbb{Z}} K=$ $\prod_{\sigma \in S} \mathbb{I}_{\sigma}$ corresponding to $\mathbb{I}_{\sigma}^{\circ}$. Regarding $P_{\tau}: \mathbb{I}_{\sigma}^{\circ} \rightarrow \overline{\mathbb{Q}}_{p}$, we have $P_{\tau}(\alpha)=\tau(\alpha)$ and $f_{P_{\tau}}=f_{P}^{\tau}$. Since $\mathbb{I}_{\sigma} \subset E\left(\widehat{\mathbf{h} \otimes_{\mathbb{Z}} K}\right)$, we have $|\tau(\alpha)|_{p}=1$. The image $a_{\sigma}(p)$ of $a(p) \otimes 1$ in $\mathbb{I}_{\sigma}$ modulo $P_{\tau}$ gives the $p$-adic unit $\tau\left(a_{P}(p)\right)$; so, $a_{\sigma}(p)$ is a unit in the integral closure of $W[[T]]$ in $\mathbb{I}_{\sigma}$.

Here is a more down-to-earth approach/proof of the fact that $\mathbb{I}_{\sigma}^{\circ}$ above gives rise to another analytic family $\mathcal{F}_{\sigma}$ containing $f_{P}^{\tau}$. Start with another arithmetic $\left(Q: \mathbb{I} \rightarrow \overline{\mathbb{Q}}_{p}\right) \in \operatorname{Spec}(\mathbb{I})\left(\overline{\mathbb{Q}}_{p}\right)$, but regarding $Q$ as a point of $\operatorname{Spec}(\mathbb{I})\left(\overline{\mathbb{Q}}_{p}\right), \mathbb{I} / Q$ has a unique embedding $\mathbb{I} / Q \subset \overline{\mathbb{Q}}_{p}$ induced by $Q: \mathbb{I} \rightarrow \overline{\mathbb{Q}}_{p}$. Then $\mathbb{I}_{\sigma}^{\circ} / Q_{\tau^{\prime}} \subset \mathbb{I} / Q \otimes_{\mathbb{Z}} K$ for the corresponding $Q_{\tau^{\prime}} \in \operatorname{Spec}\left(\mathbb{I}_{\sigma}^{\circ}\right)\left(\overline{\mathbb{Q}}_{p}\right)$. Indeed, tensoring $K$ with the exact sequence $\operatorname{Ker}(Q) \hookrightarrow \mathbb{I} \rightarrow \operatorname{Im}(Q)$, we get another exact sequence:

$$
\operatorname{Ker}(Q) \otimes_{\mathbb{Z}} K \hookrightarrow \prod_{\sigma} \mathbb{I}_{\sigma} \rightarrow \operatorname{Im}(Q) \otimes_{\mathbb{Z}} K
$$

and $\operatorname{Im}(Q) \otimes_{\mathbb{Z}} K$ contains $\sigma(K) K$ canonically and $\tau^{\prime}$ coincides with $\sigma$ on $K \cap \mathbb{Q}\left(f_{Q}\right)$ and induces $\tau^{\prime}=\left.Q_{\tau^{\prime}}\right|_{\overline{\mathbb{Q}} \cap W}: \overline{\mathbb{Q}} \cap W \hookrightarrow \overline{\mathbb{Q}}_{p}$. Then we have $f_{Q_{\tau^{\prime}}}=f_{Q}^{\tau^{\prime}}$, which is a classical modular form. It is slope 0 with respect to $i_{p}$ (i.e., with respect to the 
product topology $\left.\prod_{P} \mathcal{T}_{P}\right)$ because of $E \cdot \mathbb{I}_{\sigma}^{\circ}=\mathbb{I}_{\sigma}^{\circ}$. Thus $\mathcal{F}_{\sigma}=\left\{f_{Q}^{\tau^{\prime}}\right\}_{Q}$ is another slope 0 family. We rewrite $\sigma_{Q, \sigma}$ for $\tau^{\prime}$. Let $\pi_{\sigma}: \mathbb{I} \otimes_{\mathbb{Z}} K \rightarrow \mathbb{I}_{\sigma}^{\circ}$ be the projection. We have a commutative diagram

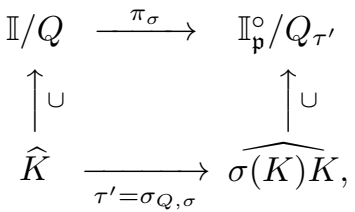

where $\widehat{K}$ is the closure of $K$ in $\mathbb{I} / Q \otimes_{\mathbb{Z}} \mathbb{Q}_{p}$ and $\widehat{\sigma(K) K}$ is the closure of $\sigma(K) K$ in $\mathbb{I}_{\sigma}^{\circ} / Q_{\tau}$.

Take $K$ to be the maximal real subfield of $M$ (to avoid complex conjugation $c$ with $\left.\left|a_{P}(p)^{c}\right|_{p}<1\right)$. Let $\Sigma$ be the CM type of $M$ given by

$$
\Sigma_{0}=\left\{\sigma: M \rightarrow \overline{\mathbb{Q}}|\sigma|_{\mathbb{Q}\left(\alpha_{0}\right)} \in \Sigma_{\alpha_{0}}\right\} .
$$

Take the starting $P$ to be $P_{0}$. Then the set $I$ of $p$-adic places of $K$ is in bijection to $\Sigma_{0, p}$, and $\left.\sigma_{P_{0}, \sigma}\right|_{M} \in \Sigma_{0}$. By the assumption (2), any prime $\mathfrak{p} \mid p$ in $K$ splits as $\mathfrak{p}=\mathfrak{P} \bar{P}$ in $M$ and $M_{\mathfrak{P}}=K_{\mathfrak{p}}=M_{\overline{\mathfrak{P}}}$; so, $M \subset \widehat{K}$ noncanonically. Since $\Sigma_{0}$ is a CM type of $M$ and since $\left\{K \hookrightarrow \sigma(K) K \mid \sigma \in \Sigma_{0}\right\}$ covers all conjugates of $K$ inside $\overline{\mathbb{Q}}$, for any $\sigma \neq \sigma^{\prime}$ in $I$, we find $P \in \mathcal{A}$ such that $\sigma_{P, \sigma}\left(\alpha_{p, P}\right) \neq \sigma_{P, \sigma^{\prime}}\left(\alpha_{p, P}\right)$ as $M$ is generated by $\left\{\alpha_{p, P}\right\}_{P \in \mathcal{A}}$. On the other hand, $\left|i_{p}\left(\sigma_{P, \sigma}\left(\alpha_{p, P_{0}}\right)\right)\right|_{p}=1$ for all $\sigma \in \Sigma_{0}$. Since any two given components of $\mathbf{h}$ do not intersect each other at any of arithmetic points (see [HMI, Proposition 3.78]), we have at least $|I|$ distinct families: $\left\{\mathcal{F}_{\sigma}\right\}_{\sigma \in I}$. In other words, the set $\Sigma_{Q}$ of $p$-adic places of $M$ induced by $\left\{\sigma_{Q, \sigma}\right\}_{\sigma \in I}$ for $Q \in \mathcal{A}$ is a $p$-adic CM type of $M$.

Since there are only finitely many $p$-adic $\mathrm{CM}$ types of $M$, replacing $\mathcal{A}$ by an infinite subset, we may assume that $\Sigma_{P}$ is identical (independent of $P$ ) for all $P \in \mathcal{A}$, and write $\Sigma_{p}$ for the set of prime ideals/places over $p$ in $K$ induced by $i_{p} \circ \sigma$ for $\sigma \in \Sigma_{P}$. This forces $\left(a_{P}(p)\right)=\prod_{\mathfrak{p} \in \Sigma_{p}^{c}} \mathfrak{p}^{e(\mathfrak{p})(k(P)-1)}$ for the absolute ramification index $e(\mathfrak{p})$ of $\mathfrak{p} /(p)$.

As before we choose an infinite sequence in $\mathcal{A}$,

$$
P_{1}, P_{2}, \ldots, P_{n}, \ldots
$$

with increasing weight $k\left(P_{1}\right)<k\left(P_{2}\right)<\cdots$ such that

$$
\left(a_{P_{j}}(p)\right)=\prod_{\mathfrak{p} \in \Sigma_{p}^{c}} \mathfrak{p}^{e(\mathfrak{p})\left(k\left(P_{j}\right)-1\right)}
$$

for all $j>0$. Then $a_{P_{j}}(p) /\langle\alpha\rangle^{k\left(P_{j}\right)-1}$ is a Weil number of weight 0 ; that is, it is an algebraic integer with all its conjugates having absolute value 1 . Then by Kronecker's theorem, we find $a_{P_{j}}(p)=\zeta_{P_{j}}\langle\alpha\rangle^{k\left(P_{j}\right)-1}$ for a root of unity $\zeta_{P_{j}}$. Note that $\langle\alpha\rangle$ is contained in a finite extension $K^{\prime} / K$. Since there are finitely many roots of unity in $K^{\prime}$, we have only finitely many possibilities for $\zeta_{P_{j}}$. Therefore, replacing $\left\{P_{j}\right\}_{j}$ by its subsequence, we find an infinite sequence $P_{1}, P_{2}, \ldots, P_{n}, \ldots$ of increasing weights such that $a_{P_{j}}(p)=\zeta\langle\alpha\rangle^{k\left(P_{j}\right)-1}$ for all $j=1,2, \ldots$ for a fixed root of unity $\zeta$. By the same argument as before, we conclude $A=\Phi_{\alpha}$, as desired.

Remark 7.1. Let $F$ be the quotient field of $W$. In the above proof, when $M$ is not an imaginary quadratic field, $\Phi_{\alpha}$ is a convergent power series with coefficients in $F$ convergent over a closed disk $D_{\epsilon}$ of radius $0<\epsilon \leq 1$ centered at an accumulation 
point $P_{\infty}$ of $\left\{P_{j} \mid j=1,2, \ldots\right\}$ (a priori, $\Phi_{\alpha}$ is not necessarily in $W[[X]]$ as we do not know if $\left.i_{p}(\alpha) \in \mathbb{Z}_{p}^{\times}\right)$. Since $r\left(P_{j}\right) \leq r$ and the $P_{j}$ s for all $j$ are inside the closed disk of radius $1-\left(1 / p^{r}(p-1)\right)$ centered at the origin, we have an accumulation point $P_{\infty}$. Replacing $\left\{P_{j}\right\}_{j}$ by an infinite subsequence, we may assume that $\left\{P_{j}\right\}_{j} \subset D_{\epsilon}$ and $\lim _{j \rightarrow \infty} P_{j}=P_{\infty}$ in $D_{\epsilon}$. Thus $\Phi_{\alpha}$ is in the power series ring $R=F\{\{t\}\}$ convergent over $D_{\epsilon}$ which is an affinoid ring over $F$. Here $t=(1+X)-P_{\infty}(1+X)$, and $\mathbb{I}$ is naturally embedded in $F\{\{t\}\}$ regarding $a \in \mathbb{I}$ as an analytic function on $D_{\epsilon}$. By the rigid analytic version of the Weierstrass preparation theorem (see [NAA, $\S 5.2 .2$ and $\S 6.1 .5]), \zeta \Phi_{\alpha}-A$ has finitely many zeros in the closed disk of radius $\epsilon$ (i.e., the rigid analytic space of $R$ ), and hence $A=\Phi_{\alpha}$ as $A_{P_{j}}=\Phi_{\alpha}\left(P_{j}\right)$ for infinitely many $j$. This then shows that $\Phi_{\alpha}$ is in the finite extension $\mathbb{I} / \Lambda$, and

hence $\Phi_{\alpha}=\zeta(1+X)^{s}$ with $s=\frac{\log _{p}(\alpha)}{\log _{p}(\gamma)}$, which has to be in $\mathbb{Q}_{p}^{\times}$(by the argument in the last paragraph of the proof of Proposition 5.2).

The following corollary shows that $M_{V, r}(\mathcal{F})(r<\infty)$ is an infinite extension if it is Steinberg (that is, its weight 2 member has abelian variety multiplicative at $p$; see [DR, $\S \mathrm{V} .3]$ and [La, Theorem 7.5]):

Corollary 7.3. Let $\mathcal{F}$ be a p-adic analytic family of classical p-ordinary Hecke eigenforms satisfying the condition (2) of the above proposition. If $\mathcal{F}$ contains a member $f_{2}$ such that $\left|a\left(p, f_{2}\right)\right|=1$, then $M_{V, r}(\mathcal{F})$ for finite $r>0$ is an infinite extension of $\mathbb{Q}$. More generally, if we find two arithmetic points $P$ with $r(P) \leq$ $r$ such that in the maximal totally real subfield of $\mathbb{Q}\left(a\left(p, f_{P}\right)\right) / \mathbb{Q}$ the prime $(p)$ decomposes differently, then $M_{V, r}(\mathcal{F})$ is an infinite extension of $\mathbb{Q}$.

Proof. By the above theorem, if $K=M_{V, r}(\mathcal{F})$ is a finite extension, then $a\left(p, f_{2}\right)$ has to be a Weil number of weight 1 , which is not the case if $f_{2}$ has multiplicative reduction. If $M_{V, r}(\mathcal{F})$ is a finite extension, then $\mathbb{Q}\left(a\left(p, f_{P}\right)\right)$ is a subfield of $M_{V, r}(\mathcal{F})$; so, the splitting of the prime $p$ in the real subfield of $M_{V, r}(\mathcal{F})$ is the same in $M_{V, r}(\mathcal{F})$. Then the second assertion is obvious.

\section{Proof of Vertical theorems}

We first prove Theorem 3.2 Suppose that $K:=M_{V, \mathcal{A}}(\mathcal{F})$ is a finite extension and assume the existence of an arithmetic point $P_{0}$ as in the theorem. Therefore the assumption (2) of Proposition 7.2 is met. By Proposition 7.2 (and the remark following its proof), we find a Weil number $\alpha$ of weight 1 and a power series $\Phi_{\alpha}(X) \in W\left[\mu_{p^{n}}\right][[X]]\left[(1+X)^{1 / p^{n}}\right]$ such that $a_{P}(p)=\Phi_{\alpha}\left(\varepsilon_{P}(\gamma) \gamma^{k(P)-1}-1\right)=$ $\zeta\left(\varepsilon_{P}(\gamma)\right)^{\log _{p}(\alpha) / \log _{p}(\gamma)}\langle\alpha\rangle^{k(P)-1}$ for all arithmetic $P$, where $\zeta$ is a root of unity independent of $P$. Then, for any infinite set $\mathcal{B}$ of arithmetic points of weight 2 , we find $M_{H, \mathcal{B}}(\mathcal{F}) \subset \mathbb{Q}\left(\mu_{p^{\infty}(p-1)}\right)(\zeta, \alpha)$, which is a finite extension of $\mathbb{Q}\left(\mu_{p^{\infty}}\right)$. Then by the strong horizontal theorem, $\mathcal{F}$ has complex multiplication. The converse follows from Corollary 4.2. This finishes the proof of Theorem 3.2 .

We now prove the vertical theorem in the introduction. We want to apply Theorem 3.2 since $M_{V, r}(\mathcal{F}) \subset \mathbb{Q}_{V, r}(\mathcal{F})$. Thus we only need to verify the assumption (2) of Proposition 7.2 to apply Theorem 3.2. Pick $P$ of weight 2 such that $A_{P}$ has good ordinary reduction modulo $\mathfrak{m}$ for the maximal ideal $\mathfrak{m}$ of $\mathbb{Z}_{p}\left[\mu_{p^{r+1}}\right]$. Since $\widetilde{A}=A_{P} \times_{\mathbb{Z}_{p}\left[\mu_{p^{r}}\right]} \mathbb{F}_{p}$ is ordinary, by a theorem of Tate (cf. [ABV], Section 22]), $\widetilde{A}$ has complex multiplication by $\mathbb{Q}(F) \subset \operatorname{End}\left(\widetilde{A} / \mathbb{F}_{p}\right)$ for the $p$-power relative Frobenius 
map $F: \widetilde{A} \rightarrow \widetilde{A}$. Note that by the congruence relation (cf. [IAT, Theorem 7.9] or GME, Section 4.2]), $F$ satisfies the characteristic polynomial

$$
T^{2}-a_{p} T+p=(T-F)(T-V)
$$

for the dual $V$ of $F$ and $a_{p}=\alpha+\alpha^{c}$ over $\mathbb{Q}\left(f_{P}\right) \subset \operatorname{End}\left(\widetilde{A} / \mathbb{F}_{p}\right) \otimes_{\mathbb{Z}} \mathbb{Q}$. Thus $\tilde{A}$ is isogenous to $\widetilde{B}^{m}\left(m=2\left[\mathbb{Q}\left(f_{P}\right): \mathbb{Q}(\alpha)\right]\right)$ for an ordinary abelian variety $\widetilde{B}_{/ \overline{\mathbb{F}}_{p}}$ with complex multiplication by $\mathbb{Q}(\alpha)$ with $\alpha$ giving $F$ on $\widetilde{B}$ (see Ch, Lemmas 3 and 6]). Take the canonical lift $B_{/ W\left(\overline{\mathbb{F}}_{p}\right)}$ of $\widetilde{B}$ over the ring of Witt vectors $W\left(\overline{\mathbb{F}}_{p}\right)$ with coefficients in $\overline{\mathbb{F}}_{p}$ (e.g., $[\mathrm{K}]$ ). Then the CM type of $B$ is given by the action of $\mathbb{Q}(\alpha)$ on $\operatorname{Lie}(B)$, which is exactly $\Sigma=\left\{\left.\sigma|| \sigma(\alpha)\right|_{p}=1\right\}$. Thus the assumption (2) of Proposition 7.2 is satisfied by $P_{0}=P$, and therefore, Theorem 3.2 implies the vertical theorem.

We could make the following conjecture, which is a vertical version of Corollary 6.3 .

Conjecture 8.1. Let $\mathcal{A} \subset \operatorname{Spec}(\mathbb{I})\left(\mathbb{C}_{p}\right)$ be an infinite set of arithmetic points $P$ with bounded level $r(P) \leq r$. Suppose that $\mathcal{F}$ does not have complex multiplication. Then we have

$$
\limsup _{P \in \mathcal{A}}\left[\mathbb{Q}\left(a\left(p, f_{P}\right)\right): \mathbb{Q}\right]=\infty \text { and } \limsup _{P \in \mathcal{A}}\left[\mathbb{Q}\left(a\left(p, f_{P}^{\circ}\right)\right): \mathbb{Q}\right]=\infty .
$$

\section{REFERENCES}

[ABV] D. Mumford, Abelian Varieties, Tata Institute of Fundamental Research Studies in Mathematics, Oxford University Press, New York, 1994. MR.2514037 (2010e:14040)

[ACM] G. Shimura, Abelian Varieties with Complex Multiplication and Modular Functions, Princeton University Press, Princeton, NJ, 1998. MR,1492449 (99e:11076)

[AME] N. M. Katz and B. Mazur, Arithmetic Moduli of Elliptic Curves, Annals of Math. Studies 108, Princeton University Press, Princeton, NJ, 1985. MR772569 (86i:11024)

[BAL] N. Bourbaki, Livre II: Algèbre, Chapitre 5, Hermann, Paris, 1959. MR0174550 (30:4751)

[Ca] H. Carayol, Sur les représentations $l$-adiques associées aux formes modulaires de Hilbert, Ann. Sci. École Norm. Sup. (4) 19 (1986), 409-468. MR870690 (89c:11083)

[Ch] C.-L. Chai, Every ordinary symplectic isogeny class in positive characteristic is dense in the moduli, Inventiones Math. 121 (1995), 439-479. MR1353306 (96f:11082)

[Ch1] C.-L. Chai, A rigidity result for $p$-divisible formal groups, Asian J. Math. 12 (2008), 193-202. MR2439259 (2009f:14089)

[Ch2] C.-L. Chai, Families of ordinary abelian varieties: canonical coordinates, $p$-adic monodromy, Tate-linear subvarieties and Hecke orbits, preprint 2003 (available at: www. math.upenn.edu/ ${ }^{\sim}$ chai).

[DR] P. Deligne and M. Rapoport, Les schémas de modules de courbes elliptiques, in "Modular functions of one variable, II (Proc. Internat. Summer School, Univ. Antwerp, Antwerp, 1972)", pp. 143-316. Lecture Notes in Math., Vol. 349, Springer, Berlin, 1973. MR0337993 (49:2762)

[GV] E. Ghate and V. Vatsal, On the local behaviour of ordinary $\Lambda$-adic representations, Ann. Inst. Fourier (Grenoble) 54 (2004), 2143-2162. MR2139691 (2006b:11050)

[GME] H. Hida, Geometric Modular Forms and Elliptic Curves, World Scientific, Singapore, 2000. MR1794402 (2001j:11022)

[H86a] H. Hida, Iwasawa modules attached to congruences of cusp forms, Ann. Sci. Ecole Norm. Sup. 4th series 19 (1986), 231-273. MR868300 (88i:11023)

[H86b] H. Hida, Galois representations into $G L_{2}\left(\mathbb{Z}_{p}[[X]]\right)$ attached to ordinary cusp forms, Inventiones Math. 85 (1986), 545-613. MR848685 (87k:11049)

[H86c] H. Hida, Hecke algebras for $G L_{1}$ and $G L_{2}$, Sém. de Théorie des Nombres, Paris 1984-85, Progress in Math. 63 (1986), 131-163. MR897346 (88i:11078)

[H88] H. Hida, On $p$-adic Hecke algebras for $G L_{2}$ over totally real fields, Ann. of Math. (2) 128 (1988), 295-384. MR 960949 (89m:11046) 
[H00] H. Hida, Adjoint Selmer groups as Iwasawa modules, Israel Journal of Math. 120 (2000), 361-427. MR.1809628 (2001k:11094)

[H10] H. Hida, The Iwasawa $\mu$-invariant of $p$-adic Hecke $L$-functions, Ann. of Math. (2) 172 (2010), 41-137.

[HM] H. Hida and Y. Maeda, Non-abelian base change for totally real fields, Olga Taussky Todd memorial issue, Pacific Journal of Math. (1997), 189-217. MR1610859 (99f:11068)

[HMI] H. Hida, Hilbert modular forms and Iwasawa theory, Oxford University Press, 2006. MR2243770 (2007h:11055)

[Ho] T. Honda, Isogeny classes of abelian varieties over finite fields, J. Math. Soc. Japan 20 (1968), 83-95. MR0229642(37:5216)

[IAT] G. Shimura, Introduction to the Arithmetic Theory of Automorphic Functions, Princeton University Press, Princeton, NJ, and Iwanami Shoten, Tokyo, 1971. MR.0314766 $(47: 3318)$

[ICF] L. C. Washington, Introduction to Cyclotomic Fields, Graduate Texts in Mathematics, 83, Springer, New York, 1982. MR1421575 (97h:11130)

[K] N. M. Katz, Serre-Tate local moduli, in "Surfaces Algébriques", Lecture Notes in Math. 868 (1978), 138-202. MR638600 (83k:14039b)

[La] R. P. Langlands, Modular forms and $\ell$-adic representations, in "Modular functions of one variable, II (Proc. Internat. Summer School, Univ. Antwerp, Antwerp, 1972)", pp. 361-500. Lecture Notes in Math., Vol. 349, Springer, Berlin, 1973. MR0354617(50:7095)

[Lo] J. H. Loxton, On two problems of R. M. Robinson about sum of roots of unity, Acta Arithmetica 26 (1974), 159-174. MR0371852(51:8069)

[M] B. Mazur, Deforming Galois representations, in "Galois group over $\mathbb{Q}$ ", MSRI publications 16 (1989), 385-437. MR 1012172 (90k:11057)

[MFG] H. Hida, Modular Forms and Galois Cohomology, Cambridge Studies in Advanced Mathematics 69, Cambridge University Press, Cambridge, England, 2000. MR:1779182 (2002b:11071)

[MFM] T. Miyake, Modular Forms, Springer, New York-Tokyo, 1989. MR.1021004 (90m:11062)

[MW] B. Mazur and A. Wiles, On $p$-adic analytic families of Galois representations, Compositio Math. 59 (1986), 231-264. MR860140 (88e:11048)

[NAA] S. Bosch, U. Güntzer and R. Remmert, Non-Archimedean analysis. A systematic approach to rigid analytic geometry. Grundlehren der Mathematischen Wissenschaften, Vol. 261, Springer, Berlin, 1984. MR746961 (86b:32031)

[R] K. Ribet, On $l$-adic representations attached to modular forms II, Glasgow Math. J. 27 (1985), 185-194. MR819838 (88a:11041)

[S] A. J. Scholl, Motives for modular forms, Inventiones Math. 100 (1990), 419-430. MR.1047142(91e:11054)

Department of Mathematics, University of California, los Angeles, Los Angeles, CALIFORNia 90095-1555

E-mail address: hida@math.ucla.edu 